\title{
Spectral Precoding for Rectangularly Pulsed OFDM
}

\author{
Char-Dir Chung, Senior Member, IEEE
}

\begin{abstract}
Spectrally precoded orthogonal frequency-division multiplexing (OFDM) is a promising rectangularly pulsed OFDM signaling format which can provide very small power spectral sidelobes, while allowing for efficient implementation by fast Fourier transform and guard interval insertion. In this paper, general constraints on spectral precoding are developed for OFDM signals with zero padding (ZP-OFDM) or cyclic prefix (CP-OFDM) to warrant the desirable spectral property that the power spectral sidelobes decay asymptotically as $f^{-2 K-2}$, where $K$ is a preassigned positive integer. In accordance with the constraints, block partitioning is adopted to construct a general signaling format to facilitate the precoder design of the spectrally precoded CP-OFDM that can provide fast decaying sidelobes. New correlative and orthogonal precoders are also devised so that the desirable spectral property of fast sidelobe decaying is achieved with spectrally precoded ZP-OFDM and CP-OFDM signals.
\end{abstract}

Index Terms-Orthogonal frequency-division multiplexing, spectral precoding, fast Fourier transform, cyclic prefix, zero padding.

\section{INTRODUCTION}

$\mathbf{O}$ RTHOGONAL frequency-division multiplexing (OFDM) has been widely adopted in modern broadband communications because of its increased robustness against channel dispersion and multipath fading [1]-[11]. Most popular OFDM approaches are standardized in the form of rectangularly pulsed OFDM block transmission that enables efficient implementation using fast Fourier transform (FFT) algorithms [12] and allows for counteracting adverse channel effects by inserting guard intervals with cyclic prefix [5]-[8] or zero padding [9]. Despite the advantages, the rectangularly pulsed OFDM signal is discontinuous in phase and exhibits relatively large power spectral sidelobes which decay asymptotically as $f^{-2}$ [11]. Although smoother pulses of finite duration [13]-[15] or infinite duration [1]-[4] have been suggested for OFDM to enhance spectral compactness, these different pulses are not practically adopted because they forbid the standard OFDM modulation by FFT and guard interval insertion.

In order to suppress sidelobe powers, frontend filtering and insertion of guard subcarriers are commonly applied to the rectangularly pulsed OFDM signal in practical transmitters [16]-[17]. However, the insertion of guard subcarriers reduces

Paper approved by J. Wang, the Editor for Wireless Spread Spectrum of the IEEE Communications Society. Manuscript received August 8, 2006; revised December 12, 2006. This work was supported by the ROC National Science Council under Contract 95-2221-E-002-088-MY2. This paper was partially presented at the IEEE International Conference on Communications, Glasgow, Scotland, June 2007.

C.-D. Chung is with the Graduate Institute of Communications Engineering and the Department of Electrical Engineering, National Taiwan University, Taipei, Taiwan 10617, Republic of China (e-mail: cdchung@cc.ee.ntu.edu.tw).

Digital Object Identifier 10.1109/TCOMM.2008.060459. the system throughout without substantially suppressing sidelobes, which still decay asymptotically as $f^{-2}$. The frontend filtering breaks the orthogonality among multiplexed subcarriers, and sometimes, consecutive OFDM blocks, and entails remarkable degradation in error performance [17].

By manipulating data blocks prior to OFDM modulation, some novel schemes have been recently proposed to suppress the sidelobe powers of the rectangularly pulsed OFDM signal, while allowing for FFT realization and guard interval insertion. There are basically two streams of sidelobe suppression schemes. One stream is based on multiplying the data symbols in frequency domain either by different weights [18], or by the same weight in conjunction with the insertion of cancellation subcarriers on spectrum borders [19]-[20]. Strikingly, the data-weighting schemes are capable of reducing sidelobe powers by more than $10 \mathrm{~dB}$. However, these schemes depend heavily on the transmitted data block in order to generate weights and/or cancellation subcarriers by numerically solving constrained optimization problems block by block, and are thus computationally intensive to realize. Moreover, the data-dependent weighting processes not only complicate the coherent demodulation on subcarriers and channel estimation, but also degrade the system error performance remarkably.

In the other stream, significant sidelobe suppression is achieved by spectrally precoding data blocks without resort to specific data values [10]-[11]. By linearly transforming each data block, spectral precoding schemes introduce correlation among data symbols in frequency domain and thus reshape the power spectrum of the rectangularly pulsed OFDM signal to exhibit very small sidelobes. These schemes apply to rectangularly pulsed OFDM with cyclic prefix (CP-OFDM) or zero padding (ZP-OFDM). The precoders proposed thus far possess structural regularity and permit implementation and analysis with ease. Particularly, correlatively $\mathcal{G}_{L^{\prime}}$-coded ZP-OFDM in [10] exhibits extremely small power spectral sidelobes decaying asymptotically as $f^{-2 L^{\prime}-2}$, where $L^{\prime}$ denotes the precoding order. Such a spectral advantage has been analytically verified by the prevailing properties of a novel function set $\{U(x ; m, n) ; m, n=0,1, \ldots\}$ proposed in [10, eq. (9)]. Because correlative coding breaks the orthogonality among multiplexed data-modulated waveforms, $\mathcal{G}_{L}$-coded OFDM degrades in error performance from uncoded OFDM on the additive white Gaussian noise (AWGN) channel and delay-spread (DS) channels with low amplitude fluctuation. Constructed from delicately designed basis sets containing full-response continuous basis signals with zero interval edges, $\mathcal{V}_{L}$ and $\mathcal{W}_{L}$-coded ZP-OFDMs and $\mathcal{V}_{L}$-coded CP-OFDM with limited guard interval lengths are developed in [11] to construct continuous-phase OFDM signals with small power spectral sidelobes decaying asymptotically as $f^{-4}$, where $L$ 
denotes the precoding order. Because the orthogonality among multiplexed data-modulated waveforms is maintained, $\mathcal{V}_{L}$ and $\mathcal{W}_{L}$-coded OFDMs prevail in average error performance over $\mathcal{G}_{L^{\prime}}$-coded OFDM on the AWGN channel and DS channels with low amplitude fluctuation.

In the paper, further results regarding spectrally precoded OFDM are presented. First, general constraints on precoder coefficients are derived for ZP-OFDM and CP-OFDM to warrant the spectral property that the power spectral sidelobes decay asymptotically as $f^{-2 K-2}$ with $K$ a preassigned positive integer. These constraints further verify the fast sidelobedecaying properties provided by the spectrally precoded OFDMs reported in [10]-[11]. Second, block partitioning is proposed in association with CP-OFDM (abbreviated as BCPOFDM) as a new signaling format to satisfy the fast sidelobedecaying constraint for CP-OFDM. New spectral precoders are devised by modifying $\mathcal{G}_{L^{\prime}}$ and $\mathcal{W}_{L}$ precoders and shown to construct the spectrally precoded BCP-OFDM signals that provide fast-decaying power spectral sidelobes. Third, based on the reduced Hadamard matrix, new precoders for ZPOFDM and BCP-OFDM are also proposed. The reduced Hadamard precoded OFDMs are shown to provide the same power spectrum as some spectrally precoded OFDMs, while requiring less implementation complexity.

\section{Spectrally Precoded OFDM: Signaling AND CONSTRAINTS}

Consider the memoryless spectrally-precoded OFDM block transmission on the nominal block duration $-T_{g}-T_{d} / 2 \leq t \leq$ $T_{d} / 2$, which consists of data block and guard intervals with lengths $T_{d}$ and $T_{g}$, respectively. On the data block interval $|t| \leq T_{d} / 2$, the rectangularly pulsed OFDM signal uses $N$ radian frequencies $\left\{\omega_{0}+n \omega_{d} ; n \in \mathcal{Z}_{N}\right\}$ and carries the transmitted complex-valued symbol block $\left\{B_{n} ; n \in \mathcal{Z}_{N}\right\}$ in the canonical form of ${ }^{1}$

$$
s(t)=\rho \operatorname{Re}\left\{\sum_{n=0}^{N-1} B_{n} \exp \left\{j\left(\omega_{0}+n \omega_{d}\right) t\right\}\right\}
$$

where $\rho$ is the signaling amplitude and $\omega_{0}$ is an integer multiple of $\omega_{d}$ with $\omega_{0} \gg \omega_{d}$ and $\omega_{d}=2 \pi / T_{d}$. On the guard interval $-T_{g}-T_{d} / 2 \leq t<-T_{d} / 2$, the transmitted signal is given by $s(t)=0$ for ZP-OFDM and $s(t)=s\left(t+T_{d}\right)$ for CP-OFDM. The complex-valued data block $\left\{D_{m} ; m \in \mathcal{Z}_{M}\right\}$ is linearly precoded to produce $\left\{B_{n} ; n \in \mathcal{Z}_{N}\right\}$ at a rate $\lambda \triangleq M / N$, as

$$
B_{n}=\sum_{m=0}^{M-1} G_{n, m} D_{m} \quad n \in \mathcal{Z}_{N}
$$

where $G_{n, m}$ s denote the complex-valued precoding coefficients. It is assumed throughout that $N$ is a positive integer power of two with $N>M$. All data symbols are modeled to be independent with zero mean and $E\left\{\left|D_{m}\right|^{2}\right\}=1$. By modeling so, $T=T_{g}+T_{d}$ denotes the OFDM block length and the data symbol time $T_{s}$ is related to $T$ by $T_{s}=T / M$.

\footnotetext{
${ }^{1}$ In the paper, we denote $\mathcal{Z}_{K} \triangleq\{0,1, \ldots, K-1\}$ and $\mathcal{Z}_{K}^{+} \triangleq\{1,2, \ldots, K\}$.
}

Using (2), the spectrally precoded OFDM signal can be alternatively expressed as

$$
s(t)=\rho \operatorname{Re}\left\{\sum_{m=0}^{M-1} D_{m} A_{m}(t)\right\}
$$

where $A_{m}(t) \triangleq \sum_{n=0}^{N-1} G_{n, m} \exp \left\{j\left(\omega_{0}+n \omega_{d}\right) t\right\}$ is defined on $|t| \leq T_{d} / 2$ and $-T_{g}-T_{d} / 2 \leq t \leq T_{d} / 2$ for ZP-OFDM and CP-OFDM, respectively. As indicated, the spectrally precoded OFDM signal consists of a multiplexing of $M$ independent component signals, each bearing a memoryless data stream through a different carrier. Using this fact, the equivalent lowpass power spectral density (PSD) of the spectrally precoded OFDM signal is obtained as

$$
\mathcal{S}_{L P}\left(f-\frac{\omega_{0}}{2 \pi}-\frac{(N-1) \omega_{d}}{4 \pi}\right)=\frac{\rho^{2}}{2 T} \sum_{m=0}^{M-1}\left|\mathcal{F}\left\{A_{m}(t)\right\}\right|^{2}
$$

with $\mathcal{F}\{\cdot\}$ denoting Fourier transform, and specializes for $\mathrm{CP}$ OFDM and ZP-OFDM to [11]

$$
\begin{aligned}
\mathcal{S}_{L P}^{C P}(f)= & \frac{\rho^{2} T}{2} \sum_{m=0}^{M-1} \mid \sum_{n=0}^{N-1} G_{n, m} \exp \left\{-j \frac{n}{2} \omega_{d} T_{g}\right\} \\
& \left.\cdot \operatorname{sinc}\left(\left(n-\frac{N-1}{2}\right) \frac{T}{T_{d}}-f T\right)\right|^{2} \\
\mathcal{S}_{L P}^{Z P}(f)= & \frac{\rho^{2} T_{d}^{2}}{2 T} \sum_{m=0}^{M-1} \\
& \left|\sum_{n=0}^{N-1} G_{n, m} \operatorname{sinc}\left(n-\frac{N-1}{2}-f T_{d}\right)\right|^{2}
\end{aligned}
$$

with $\operatorname{sinc}(x) \triangleq \sin (\pi x) /(\pi x)$. The average signal power $P$ is related to $\rho$ by $P=\frac{\rho^{2}}{2} \sum_{m=0}^{M-1}$ $\sum_{n=0}^{N-1} \sum_{l=0}^{N-1} G_{n, m} G_{l, m}^{*} \exp \left\{j \frac{l-n}{2} \omega_{d} T_{g}\right\} \operatorname{sinc}\left((n-l) \frac{T}{T_{d}}\right)$ for CP-OFDM and $P=\frac{\rho^{2} T_{d}}{2 T} \sum_{m=0}^{M-1} \sum_{n=0}^{N-1}\left|G_{n, m}\right|^{2}$ for ZP-OFDM.

Because $s(t)$ in (1) is constituted by the multiplexing of rectangularly-pulsed $\omega_{d}$-spaced complex exponentials, it can be realized by the standard OFDM process in conjunction with spectral precoding. Fig. 1 depicts the spectrally precoded OFDM system. As shown, each data block $\left\{D_{m} ; m \in \mathcal{Z}_{M}\right\}$ is first precoded by (2). Its output block $\left\{B_{n} ; n \in \mathcal{Z}_{N}\right\}$ is then fed into the standard inverse-FFT-based OFDM transmission process to generate $s(t)$. For reference convenience, coefficients for some precoders presented herein are summarized in Table I. By appropriately constraining $G_{n, m} \mathrm{~s}$, spectrally precoded OFDM signals can be designed to exhibit desirable signaling characteristics, as follows.

A) Orthogonality Constraint: If we place the orthogonality constraint on $G_{n, m} \mathrm{~s}$,

$$
\sum_{n=0}^{N-1} G_{n, m} G_{n, l}^{*}=\delta_{m, l} \quad m, l \in \mathcal{Z}_{M}
$$

with $\delta_{m, l}$ defined by $\delta_{m, l}=1$ if $m=l$ and $\delta_{m, l}=0$ otherwise, $\left\{A_{m}(t) ;|t| \leq T_{d} / 2\right\}_{m=0}^{M-1}$ forms a unit-power complex basis signal set. ${ }^{2}$ For convenience, the precoder satisfying

${ }^{2}$ Here, $A_{m}(t) \mathrm{s}$ satisfy $\int_{-T_{d} / 2}^{T_{d} / 2} A_{m}(t) A_{n}(t) d t=0$ and $\int_{-T_{d} / 2}^{T_{d} / 2}$ $A_{m}(t) A_{n}^{*}(t) d t=T_{d} \delta_{m, n}$ for all $m, n \in \mathcal{Z}_{M}$. 


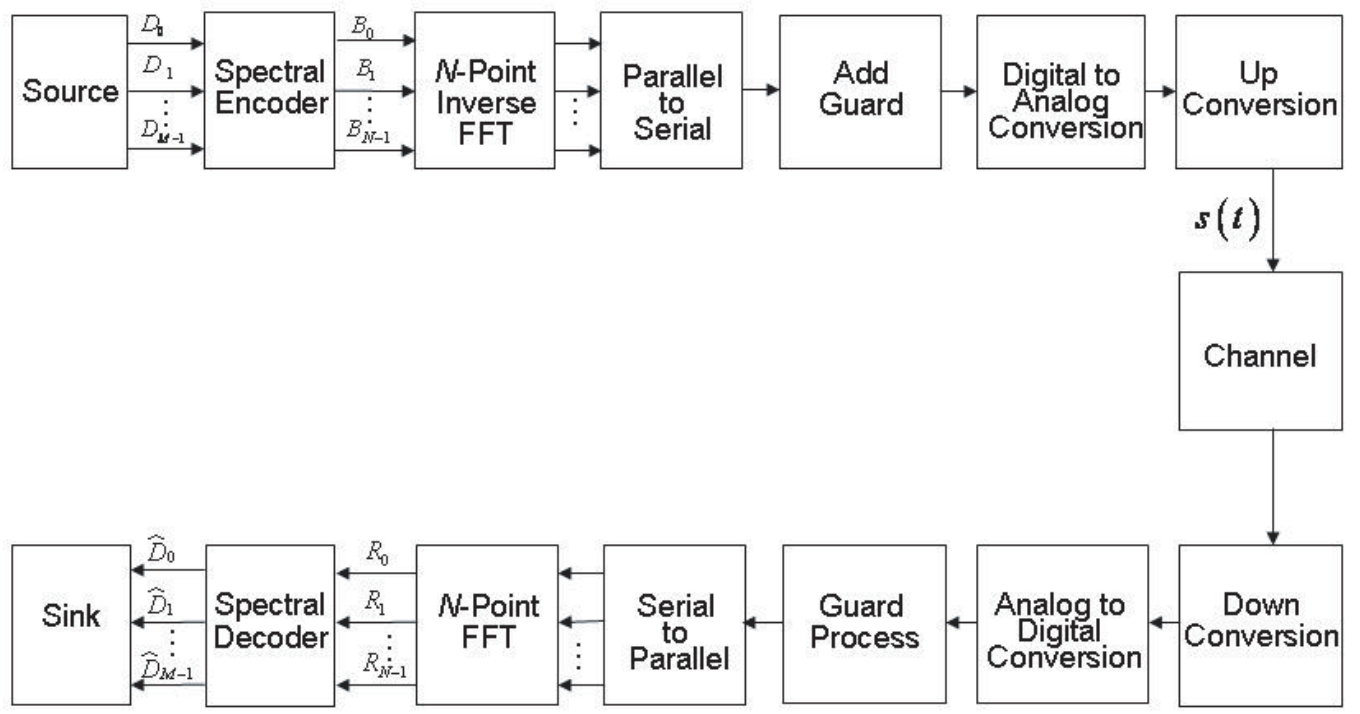

Fig. 1. Spectrally precoded OFDM system.

TABLE I

Characterization of $\mathcal{G}_{L}, \mathcal{H}_{L}, \mathcal{V}_{L}, \mathcal{W}_{L}, \mathcal{G}_{I, L}, \mathcal{H}_{I, L}$, AND $\mathcal{W}_{I, L}$ PRecoders. Precoders $\mathcal{G}_{L}, \mathcal{H}_{L}, \mathcal{V}_{L}$, And $\mathcal{W}_{L}$ Can be applied to ZP-OFDM AND CP-OFDM With ARBITRARY $T_{g} / T_{d}$ RATIOS. PRECODERs $\mathcal{G}_{I, L}, \mathcal{H}_{I, L}$, AND $\mathcal{W}_{I, L}$ ARE RESTRICTED FOR USE IN BCP-OFDM WITH $T_{g}=2^{-I} T_{d}$.

\begin{tabular}{|c|c|}
\hline Precoder & Coefficients \\
\hline $\begin{array}{c}\mathcal{G}_{L} \\
\left(\lambda=1-\frac{L}{N}, L=N-M\right)\end{array}$ & $\begin{array}{ll}G_{m+l, m}=\zeta_{m+l}\left(\begin{array}{l}L \\
l\end{array}\right), & l \in \mathcal{Z}_{L+1} \text { and } m \in \mathcal{Z}_{M} \\
G_{n, m}=0, & \text { otherwise } \\
\end{array}$ \\
\hline $\begin{array}{c}\mathcal{H}_{L} \\
\left(\lambda=1-2^{-L}, L \in \mathcal{Z}_{\log _{2} N}^{+}\right)\end{array}$ & $\begin{array}{ll}G_{n 2^{L}+\alpha, n\left(2^{L}-1\right)+\beta-1}=2^{-\frac{L}{2}}(-1)^{\alpha} h_{\alpha, \beta}, & n \in \mathcal{Z}_{2^{-L} N}, \alpha \in \mathcal{Z}_{2^{L}}, \beta \in \mathcal{Z}_{2^{L}-1}^{+} \\
G_{n, m}=0, & \text { otherwise } \\
\end{array}$ \\
\hline $\begin{array}{c}\mathcal{V}_{L} \\
\left(\lambda=1-2^{-L}, L \in \mathcal{Z}_{\log _{2} N}^{+}\right)\end{array}$ & $\begin{array}{ll}G_{n+v 2^{-u} N, \tilde{\chi}(u)+n}=2^{-\frac{u}{2}}(-1)^{\phi_{u, v}}, & n \in \mathcal{Z}_{2^{-u} N}, v \in \mathcal{Z}_{2^{u}}, \text { and } u \in \mathcal{Z}_{L}^{+} \\
G_{n, m}=0, & \text { otherwise }\end{array}$ \\
\hline $\begin{array}{c}\mathcal{W}_{L} \\
\left(\lambda=1-2^{-L}, L \in \mathcal{Z}_{\log _{2} N}^{+}\right)\end{array}$ & $\begin{array}{ll}G_{n 2^{u}+v, \widetilde{\chi}(u)+n}=2^{-\frac{u}{2}}(-1)^{\psi_{u, v}}, & n \in \mathcal{Z}_{2^{-u} N}, v \in \mathcal{Z}_{2^{u}}, \text { and } u \in \mathcal{Z}_{L}^{+} \\
G_{n, m}=0, & \text { otherwise }\end{array}$ \\
\hline $\begin{array}{c}\mathcal{G} I, L \\
\left(\lambda=1-\frac{L}{2^{-I} N}, L=2^{-I}(N-M)\right)\end{array}$ & $\begin{array}{ll}g_{m+l, m}=(-1)^{m+l}\left(\begin{array}{l}L \\
l\end{array}\right), & l \in \mathcal{Z}_{L+1} \text { and } m \in \mathcal{Z}_{2^{-I} M} \\
g_{n, m}=0, & \text { otherwise } \\
\end{array}$ \\
\hline $\begin{array}{c}\mathcal{H}_{I, L} \\
\left(\lambda=1-2^{-L}, L \in \mathcal{Z}_{\log _{2} N-I}^{+}\right)\end{array}$ & $\begin{array}{ll}g_{n 2^{L}+\alpha, n\left(2^{L}-1\right)+\beta-1}=2^{-\frac{L}{2}} h_{\alpha, \beta}, & n \in \mathcal{Z}_{2-I-L_{N}}, \alpha \in \mathcal{Z}_{2^{L}}, \beta \in \mathcal{Z}_{2^{L}-1}^{+} \\
g_{n, m}=0, & \text { otherwise }\end{array}$ \\
\hline $\begin{array}{c}\mathcal{W}_{I, L} \\
\left(\lambda=1-2^{-L}, L \in \mathcal{Z}_{\log _{2} N-I}^{+}\right)\end{array}$ & $\begin{array}{ll}g_{n 2^{u}+v, \chi(u)+n}=2^{-\frac{u}{2}}(-1)^{v_{u}}, & n \in \mathcal{Z}_{2^{-I-u} N}, v \in \mathcal{Z}_{2^{u}}, \text { and } u \in \mathcal{Z}_{L}^{+} \\
g_{n, m}=0, & \text { otherwise }\end{array}$ \\
\hline
\end{tabular}

The coefficients $\zeta_{n}, \phi_{u, v}, \psi_{u, v}, \tilde{\chi}(u)$ and $\chi(u)$ are defined as follows: (a) $\zeta_{n}=\exp \left\{j \frac{n}{2} \omega_{d} T_{g}\right\}$ for $\mathcal{G}_{L}$-coded CP-OFDM and $\zeta_{n}=1$ for $\mathcal{G}_{L}$-coded ZP-OFDM. (b) $\phi_{u, v}=v_{1}$ for $u<\log _{2} N$ and $\phi_{\log _{2} N, v}=0$. (c) $\psi_{u, v}=1+v_{1}+v_{u}$ for $u \geq 2$ and $\psi_{1, v}=0$. (d) $\tilde{\chi}(u)=N\left(1-2^{1-u}\right.$ ). (e) $\chi(u)=2^{-I} N\left(1-2^{1-u}\right)$. Here, $\left(v_{1}, v_{2}, \ldots, v_{u}\right)$ denotes the binary representation of $v$, i.e., $v=\sum_{l=1}^{u} v_{l} 2^{l-1}$ with $v_{l} \in \mathcal{Z}_{2}$.

(7) is called orthogonal. For example, $\mathcal{W}_{L}$ and $\mathcal{V}_{L}$ precoders in [11] are orthogonal. Under the constraint, $s(t)$ in (3) consists of the orthogonal multiplexing of $M$ data-modulated waveforms on the data block interval, and $\left\{D_{m} ; m \in \mathcal{Z}_{M}\right\}$ can be decoded from $\left\{B_{n} ; n \in \mathcal{Z}_{N}\right\}$ by

$$
D_{m}=\sum_{n=0}^{N-1} G_{n, m}^{*} B_{n}, \quad m \in \mathcal{Z}_{M} .
$$

B) Fast Sidelobe-Decaying Constraints: In the nominal block duration, $s(t)$ is continuous and has continuous derivatives for all orders inside the rectangular window, i.e., $-T_{d} / 2-T_{g}<t<T_{d} / 2$ for CP-OFDM and $|t|<T_{d} / 2$ for ZP-OFDM. However, $s(t)$ may not exhibit continuity at the window edges, depending on $G_{n, m} \mathrm{~s}$. When $s(t)$ is discontinuous at window edges (e.g., uncoded CP-OFDM and ZP-OFDM), $\mathcal{S}_{L P}(f)$ provides large power spectral sidelobes which roll off slowly as $f^{-2}[10]-[11]$. When $s(t)$ is contin- uous at window edges and thus has continuous phase [11], $\mathcal{S}_{L P}(f)$ provides small sidelobes which roll off as $f^{-4}$ or faster. As shown in [11], such phase continuity exists for $\mathcal{V}_{L}$ and $\mathcal{W}_{L}$-coded ZP-OFDM and $\mathcal{V}_{L}$-coded CP-OFDM with $T_{g} / T_{d} \in\left\{2^{-1}, 2^{-2}, \ldots, 2^{-\left\{\log _{2} N-L\right)}\right\}$. Here, general constraints on $G_{n, m}$ s are developed for ZP-OFDM and CPOFDM to enable continuity on window edges and thereby guarantee fast-decaying spectral sidelobes.

Consider spectrally precoded ZP-OFDM first. If the precoder satisfies the constraint ${ }^{3}$

$$
\text { Constraint 1: } \begin{aligned}
& \sum_{n=0}^{N-1}(-1)^{n} n^{l} G_{n, m}=0 \text { for } l \in \mathcal{Z}_{K} \\
& \text { and } \sum_{n=0}^{N-1}(-1)^{n} n^{K} G_{n, m} \neq 0
\end{aligned}
$$

${ }^{3}$ By default, $0^{0} \equiv 1$. 
for a positive integer $K$ and all $m \in \mathcal{Z}_{M}$, then the identity $\left.\frac{d^{l}}{d t^{l}} A_{m}(t)\right|_{t= \pm T_{d} / 2}=0$ holds for all $l \in \mathcal{Z}_{K}$ and $m \in \mathcal{Z}_{M}$. Thus, the corresponding ZP-OFDM signal has continuous derivatives up to the $(K-1)$ th order at all times and for all data blocks (see (3)). Moreover, $\left|\mathcal{F}\left\{A_{m}(t)\right\}\right|^{2}$ in (4) decays asymptotically as $f^{-2 K-2}[21]$, and so does $\mathcal{S}_{L P}^{Z P}(f)$. This property can be further verified as follows. With $z \triangleq f T_{d}+(N-1) / 2$, $\mathcal{S}_{L P}^{Z P}(f)$ in (6) is rewritten as ${ }^{4}$

$\mathcal{S}_{L P}^{Z P}(f)=\frac{\rho^{2} T_{d}^{2}}{2 \pi^{2} T} \sin ^{2}(\pi z) \sum_{m=0}^{M-1}\left|\sum_{n=0}^{N-1}(-1)^{n} G_{n, m}(n-z)^{-1}\right|^{2}$

which can be expanded by the geometric series [22, eq. $1.112 .1]$ as

$$
\begin{aligned}
\mathcal{S}_{L P}^{Z P}(f)= & \frac{\rho^{2} T_{d}^{2}}{2 \pi^{2} z^{2} T} \sin ^{2}(\pi z) \\
& \cdot \sum_{m=0}^{M-1}\left|\sum_{l=0}^{\infty} z^{-l} \sum_{n=0}^{N-1}(-1)^{n} n^{l} G_{n, m}\right|^{2} .
\end{aligned}
$$

for $|z|>N-1$. Thus, if Constraint 1 is met, $\mathcal{S}_{L P}^{Z P}(f)$ in (10) decays asymptotically with $f^{-2 K-2}$ as $|f|$ approaches infinity. This gives the proposition.

Proposition 1: If $G_{n, m}$ s satisfy Constraint 1 , spectrally precoded ZP-OFDM provides small power spectral sidelobes decaying asymptotically as $f^{-2 K-2}$.

By investigating the precoder coefficients in Table I, it is straightforward to show that correlatively $\mathcal{G}_{L}$-coded ZPOFDM in [10] follows Proposition 1 for $K=L$, and also that orthogonally $\mathcal{W}_{L}$ and $\mathcal{V}_{L}$-coded ZP-OFDMs in [11] follow Proposition 1 for $K=1$.

Next, consider spectrally precoded CP-OFDM with $T_{g}=$ $2^{-I} T_{d}$ for $I \in \mathcal{Z}_{\log _{2} N-1}^{+}$and precoder coefficients $G_{n, m} \mathrm{~s}$ satisfying the constraint

Constraint 2a: For a given $m \in \mathcal{Z}_{M}, G_{n, m}$ is nonzero only when $n=\varpi 2^{I}$

$+\varphi(m)$ for all $\varpi \in \mathcal{Z}_{2^{-I} N}$, with $\varphi(m) \in \mathcal{Z}_{2^{I}}$ depending on $m$.

In the case, $\mathcal{S}_{L P}^{C P}(f)$ in (5) can be simplified to

$$
\begin{aligned}
\mathcal{S}_{L P}^{C P}(f)= & \frac{\rho^{2} T}{2 \pi^{2}} \sum_{m=0}^{M-1} \sin ^{2}(\pi z(m)) \mid \sum_{\varpi=0}^{2^{-I} N-1} G_{\varpi 2^{I}+\varphi(m), m} \\
& \left.\cdot\left(\varpi\left(2^{I}+1\right)-z(m)\right)^{-1}\right|^{2}
\end{aligned}
$$

with $z(m) \triangleq f T+[(N-1) / 2-\varphi(m)]\left(1+2^{-I}\right)$. When $|z(m)|>\left(2^{-I} N-1\right)\left(2^{I}+1\right)$ for all $m \in \mathcal{Z}_{M}, \mathcal{S}_{L P}^{C P}(f)$ can be further expanded using [22, eq. 1.112.1] as

$$
\begin{aligned}
\mathcal{S}_{L P}^{C P}(f)= & \frac{\rho^{2} T}{2 \pi^{2}} \sum_{m=0}^{M-1} \sin ^{2}(\pi z(m)) \mid \sum_{l=0}^{\infty}\left(2^{I}+1\right)^{l} z(m)^{-l-1} \\
& \left.\cdot \sum_{\varpi=0}^{2^{-I}} \varpi^{l} G_{\varpi 2^{I}+\varphi(m), m}\right|^{2}
\end{aligned}
$$

${ }^{4}$ For orthogonally precoded ZP-OFDM, $P=\rho^{2} M T_{d} /(2 T)$.
If $G_{n, m} \mathrm{~s}$ further satisfy

$$
\begin{aligned}
\text { Constraint } 2 b: \sum_{\varpi=0}^{2^{-I} N-1} \varpi^{l} G_{\varpi 2^{I}+\varphi(m), m}=0 \text { for } l \in \mathcal{Z}_{K} \\
\text { and } \sum_{\varpi=0}^{2^{-I} N-1} \varpi^{K} G_{\varpi 2^{I}+\varphi(m), m} \neq 0
\end{aligned}
$$

for a positive integer $K$ and all $m \in \mathcal{Z}_{M}$, then $\mathcal{S}_{L P}^{C P}(f)$ in (12) decays asymptotically with $f^{-2 K-2}$ as $|f|$ approaches infinity. Moreover, under Constraint $2 b$, the identity $\frac{d^{l}}{d t} A_{m}(t)$

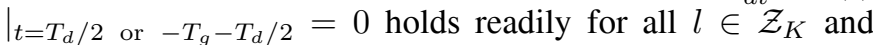
$m \in \mathcal{Z}_{M}$. Thus, the corresponding CP-OFDM signal has continuous derivatives up to the $(K-1)$ th order at all times and for all data blocks (see (3)). This yields the following proposition.

Proposition 2: If $G_{n, m} \mathrm{~s}$ satisfy Constraints $2 a$ and $2 b$, spectrally precoded CP-OFDM with $T_{g}=2^{-I} T_{d}$ for $I \in$ $\mathcal{Z}_{\log _{2} N-1}^{+}$provides small power spectral sidelobes decaying asymptotically as $f^{-2 K-2}$.

Note that $\mathcal{V}_{L}$-coded CP-OFDM with $T_{g} / T_{d} \in$ $\left\{2^{-1}, 2^{-2}, \ldots, 2^{-\left\{\log _{2} N-L\right)}\right\} \quad[11]$ follows Proposition 2 for $K=1$. More CP-OFDM schemes following Proposition 2 are exploited below.

\section{Spectrally Precoded CP-OFDM With Block PARTITIONING}

Here, a new signaling format BCP-OFDM is formulated to follow Proposition 2 by associating spectrally precoded CPOFDM with block partition. Specifically, consider the BCPOFDM signal which adopts $T_{g}=2^{-I} T_{d}$ for $I \in \mathcal{Z}_{\log _{2} N-1}^{+}$ and assigns $N$ and $M$ to be both divisible by $2^{I}$. Both symbol blocks $\left\{D_{m} ; m \in \mathcal{Z}_{M}\right\}$ and $\left\{B_{n} ; n \in \mathcal{Z}_{N}\right\}$ are evenly partitioned into $2^{I}$ subblocks, each containing uniformly spaced symbols, as

$$
\begin{aligned}
\left\{D_{m} ; m \in \mathcal{Z}_{M}\right\} & =\bigcup_{i=0}^{2^{I}-1}\left\{D_{i+m 2^{I}} ; m \in \mathcal{Z}_{2^{-I} M}\right\} \\
\left\{B_{n} ; n \in \mathcal{Z}_{N}\right\} & =\bigcup_{i=0}^{2^{I}-1}\left\{B_{i+n 2^{I}} ; n \in \mathcal{Z}_{2^{-I} N}\right\}
\end{aligned}
$$

Each data symbol subblock is precoded by the identical set of subblock coefficients so that $G_{n, m} \mathrm{~s}$ can be specified by $G_{i+n 2^{I}, i+m 2^{I}}=g_{n, m}$ for $i \in \mathcal{Z}_{2^{I}}, n \in \mathcal{Z}_{2^{-I} N}$ and $m \in$ $\mathcal{Z}_{2^{-I} M}$, and $G_{n, m}=0$ otherwise. Such precoder coefficients satisfy Constraint $2 a$ and thus reduce (2) to

$B_{i+n 2^{I}}=\sum_{m=0}^{2^{-I} M-1} g_{n, m} D_{i+m 2^{I}}, \quad n \in \mathcal{Z}_{2^{-I} N}$ and $i \in \mathcal{Z}_{2^{I}}$.

Using (13) in (1) constitutes the spectrally precoded BCPOFDM signal. The equivalent lowpass PSD $\mathcal{S}_{L P}^{B C P}(f)$ can be 
obtained from (5) as

$$
\begin{aligned}
\mathcal{S}_{L P}^{B C P}(f)= & \frac{\rho^{2} T}{2} \sum_{i=0}^{2^{I}-1} \sum_{m=0}^{2^{-I} M-1} \mid \sum_{n=0}^{2^{-I} N-1} g_{n, m}(-1)^{n} \\
& \left.\cdot \operatorname{sinc}\left(n\left(2^{I}+1\right)-z_{i}\right)\right|^{2} \\
= & \frac{\rho^{2} T}{2 \pi^{2}} \sum_{i=0}^{2^{I}-1} \sin ^{2}\left(\pi z_{i}\right) \sum_{m=0}^{2^{-I} M-1} \\
& \left|\sum_{n=0}^{2^{-I} N-1} g_{n, m}\left[n\left(2^{I}+1\right)-z_{i}\right]^{-1}\right|^{2}
\end{aligned}
$$

where $z_{i} \triangleq f T+[(N-1) / 2-i]\left(1+2^{-I}\right)$ and $P=$ $\rho^{2} 2^{I-1} \sum_{m=0}^{2^{-I} M-1} \sum_{n=0}^{2^{-I} N-1}\left|g_{n, m}\right|^{2}$. When $\left|z_{i}\right|>\left(2^{-I} N-\right.$ 1) $\left(2^{I}+1\right)$ for all $i \in \mathcal{Z}_{2^{I}}, \mathcal{S}_{L P}^{B C P}(f)$ can be expanded from (15) as

$$
\begin{aligned}
\mathcal{S}_{L P}^{B C P}(f)= & \frac{\rho^{2} T}{2 \pi^{2}} \sum_{i=0}^{2^{I}-1} z_{i}^{-2} \sin ^{2}\left(\pi z_{i}\right) \sum_{m=0}^{2^{-I} M-1} \\
& \left|\sum_{l=0}^{\infty}\left(2^{I}+1\right)^{l} z_{i}^{-l} \sum_{n=0}^{2^{-I} N-1} n^{l} g_{n, m}\right|^{2} .
\end{aligned}
$$

Further, if $G_{n, m} \mathrm{~s}$ satisfy Constraint $2 b$ or, equivalently, if $g_{n, m}$ s satisfy

$$
\begin{gathered}
\text { Constraint } 2 c: \quad \sum_{n=0}^{2^{-I} N-1} n^{l} g_{n, m}=0 \text { for } l \in \mathcal{Z}_{K} \\
\text { and } \sum_{n=0}^{2^{-I}} n^{K} g_{n, m} \neq 0
\end{gathered}
$$

for a positive integer $K$ and all $m \in \mathcal{Z}_{2^{-I} M}$, the spectrallyprecoded BCP-OFDM has continuous derivatives up to the $(K-1)$ th order at all times and for all data blocks, and provides power spectral sidelobes decaying asymptotically as $|f|^{-2 K-2}$ pursuant to Proposition 2.

Moreover, if $G_{n, m} \mathrm{~s}$ satisfy (7) or, equivalently, if $g_{n, m} \mathrm{~s}$ satisfy

$$
\sum_{n=0}^{2^{-I} N-1} g_{n, m} g_{n, l}^{*}=\delta_{m, l}, \quad m, l \in \mathcal{Z}_{2^{-I} M}
$$

a precoder for BCP-OFDM is orthogonal, and $D_{m} \mathrm{~s}$ can be decoded from $B_{n}$ s by

$D_{i+m 2^{I}}=\sum_{n=0}^{2^{-I} N-1} g_{n, m}^{*} B_{i+n 2^{I}}, \quad m \in \mathcal{Z}_{2^{-I} M}$ and $i \in \mathcal{Z}_{2^{I}}$

Three new precoders are proposed below for BCP-OFDM with $T_{g}=2^{-I} T_{d}$ for $I \in \mathcal{Z}_{\log _{2} N-1}^{+}$. The first is a correlative precoder modified from $\mathcal{G}_{L}$ and thus denoted by $\mathcal{G}_{I, L}$. The other two precoders are orthogonal and denoted by $\mathcal{U}_{I, L}$ and $\mathcal{W}_{I, L}$, where $\mathcal{U}_{I, L}$ is obtained by orthogonalizing the coefficients of $\mathcal{G}_{I, L}$ and $\mathcal{W}_{I, L}$ is modified from $\mathcal{W}_{L}$.

A) $\mathcal{G}_{I, L}$-coded BCP-OFDM: For $L \in \mathcal{Z}_{2^{-I} N-1}^{+}$, the $L$ thorder precoder $\mathcal{G}_{I, L}$ is defined as

$$
g_{n, m}=(-1)^{n}\left(\begin{array}{c}
L \\
n-m
\end{array}\right), \quad n \in\{m, m+1, \ldots, m+L\}
$$

and $g_{n, m}=0$ otherwise where $L=2^{-I}(N-M)$. Quoting [22, eq. 0.154.6], these $g_{n, m} \mathrm{~s}$ can be shown to satisfy Constraint $2 c$ with $K=L$. Thus, according to Proposition $2, \mathcal{G}_{I, L^{-}}$ coded BCP-OFDM provides small power spectral sidelobes decaying asymptotically as $|f|^{-2 L-2}$.

If we define a new real-valued function $U_{J}(x ; m, n)$ for any real $x$, nonnegative integers $m$ and $n$, and positive odd integer $J$ by

$$
U_{J}(x ; m, n) \triangleq \pi^{-1} m ! J^{m} \prod_{k=0}^{m}[(n+k) J-x]^{-1} \sin (\pi(n J-x))
$$

then the equivalent lowpass PSD of $\mathcal{G}_{I, L}$-coded BCP-OFDM can be simplified from (14) as

$$
\left.\mathcal{S}_{L P}^{B C P}(f)\right|_{\mathcal{G}_{I, L}}=\frac{P T}{M\left(\begin{array}{c}
2 L \\
L
\end{array}\right)} \sum_{i=0}^{2^{I}-1} \sum_{m=0}^{2^{-I} M-1} U_{2^{I}+1}^{2}\left(z_{i} ; L, m\right)
$$

(see Appendix A). Because $U_{2^{I}+1}^{2}\left(z_{i} ; L, m\right)$ varies proportionally with $f^{-2 L-2}$ as $|f|$ is large, so does $\left.\mathcal{S}_{L P}^{B C P}(f)\right|_{\mathcal{G}_{I, L}}$. This further verifies the fast sidelobe decaying property of $\mathcal{G}_{I, L^{-}}$ coded BCP-OFDM.

B) $\mathcal{U}_{I, L}$-coded BCP-OFDM: Consider $L \in \mathcal{Z}_{2^{-I} N-1}^{+}$and $L=2^{-I}(N-M)$. Now, define the column vector $\underline{\mu}_{m} \triangleq$ $\left[\mu_{0, m}, \mu_{1, m}, \ldots, \mu_{2^{-I} N-1, m}\right]^{t}$ for $m \in \mathcal{Z}_{2^{-I} M}$, with entries $\mu_{n, m} \mathrm{~s}$ given by $\mu_{n, m}=(-1)^{n}\left(\begin{array}{c}L \\ n-m\end{array}\right)$ for $n \in\{m, m+$ $1, \ldots, m+L\}$ and $\mu_{n, m}=0$ otherwise. ${ }^{5}$ We note that $\mu_{n, m} \mathrm{~s}$ are exactly the precoder coefficients for $\mathcal{G}_{I, L}$ and that, when stacked in a $2^{-I} N$-by-2 $2^{-I} M$ matrix, $\left[\underline{\mu}_{0}, \underline{\mu}_{1}, \ldots, \underline{\mu}_{2-I} M-1\right]$ forms a $2^{-I} N$-by-2 $2^{-I} M$ upper triangular matrix which has rank $2^{-I} M$. Hence, $\underline{\mu}_{0}, \underline{\mu}_{1}, \ldots, \underline{\mu}_{2^{-I} M-1}$ are linearly independent and can be linearly transformed to form a set of $2^{-I} M$ orthonormal vectors. Invoking the Gram-Schmidt orthogonalization process [23], such an orthonormal set can be obtained as $\underline{g}_{0}, \underline{g}_{1}, \ldots, \underline{g}_{2^{-I} M-1}$ where $\underline{g}_{m} \triangleq\left[g_{0, m}, g_{1, m}, \ldots, g_{2^{-I} N-1, m}\right]^{t}$ is obtained from $\underline{g}_{m}=\underline{w}_{m} /\left\|\underline{w}_{m}\right\|$ via the recursion

$$
\underline{w}_{m}=\underline{\mu}_{m}-\sum_{l=0}^{m-1} \frac{\underline{\mu}_{m}^{t} \underline{w}_{l}}{\left\|\underline{w}_{l}\right\|^{2}} \underline{w}_{l}, \quad m \in \mathcal{Z}_{2^{-I} M}^{+}
$$

with $\underline{w}_{0}=\underline{\mu}_{0}{ }^{6}{ }^{6}$ The $L$ th-order precoder $\mathcal{U}_{I, L}$ is thus defined by vector entries $g_{n, m} \mathrm{~s}$. Because $\underline{g}_{0}, \underline{g}_{1}, \ldots, \underline{g}_{2^{-I} M-1}$ are orthonormal and linear combinations of $\underline{\mu}_{0}, \underline{\mu}_{1}, \ldots, \underline{\mu}_{2^{-I} M-1}$ with $\mu_{n, m} \mathrm{~s}$ satisfying Constraint $2 c$ with $\bar{K}=\bar{L}, g_{n, m} \mathrm{~s}$ satisfy Constraint $2 c$ with $K=L$ and (17). Thus, $\mathcal{U}_{I, L}$ is an orthogonal precoder with rate $\lambda=1-\frac{L}{2^{-I} N}$ and $\mathcal{U}_{I, L^{-}}$ coded BCP-OFDM provides small power spectral sidelobes decaying asymptotically as $f^{-2 L-2}$.

Although (22) is formulated for $I \in \mathcal{Z}_{\log _{2} N-1}^{+}$, it also holds for $I=0$. When $I=0$, a new orthogonal precoder $\mathcal{U}_{L}$ for $\mathrm{ZP}$ OFDM can be defined by making $G_{n, m}=g_{n, m}$ for $n \in \mathcal{Z}_{N}$ and $m \in \mathcal{Z}_{M}$ where $g_{n, m} \mathrm{~s}$ are obtained from (22) by setting $\mu_{n, m}=\left(\begin{array}{c}L \\ n-m\end{array}\right)$ for $n \in\{m, m+1, \ldots, m+L\}$ and $\mu_{n, m}=$ 0 otherwise. Likewise, $\mathcal{U}_{L}$-coded ZP-OFDM provides small power spectral sidelobes decaying asymptotically as $f^{-2 L-2}$.

\footnotetext{
${ }^{5} \underline{x}^{t}$ and $\|\underline{x}\|$ denote the transpose and the norm, respectively, of $\underline{x}$.

${ }^{6}$ Because $\mu_{n, m}$ is nonzero only when $m \leq n \leq m+L, g_{n, m}=0$ for $n>m+L$. This is useful when realizing $\mathcal{U}_{I, L}$.
} 
C) $\mathcal{W}_{I, L}$-coded BCP-OFDM: For $L \in \mathcal{Z}_{\log _{2} N-I}^{+}$, the $L$ thorder precoder $\mathcal{W}_{I, L}$ is defined as

$$
g_{n 2^{u}+v, \chi(u)+n}=2^{-\frac{u}{2}}(-1)^{v_{u}}, \quad n \in \mathcal{Z}_{2^{-I-u} N}, v \in \mathcal{Z}_{2^{u}}
$$

for $u \in \mathcal{Z}_{L}^{+}$and $g_{n, m}=0$ otherwise, where $\chi(u)$ and $v_{u}$ are as defined in Table I. In the case, $M=N\left(1-2^{-L}\right)$. After some algebra, $g_{n, m} \mathrm{~s}$ can be shown to satisfy Constraint $2 c$ with $K=1$ and (17). Therefore, $\mathcal{W}_{I, L}$ is orthogonal and $\mathcal{W}_{I, L^{-}}$ coded BCP-OFDM provides small power spectral sidelobes decaying asymptotically as $f^{-4}$. Further, using (23) in (15) gives

$$
\begin{aligned}
\left.\mathcal{S}_{L P}^{B C P}(f)\right|_{\mathcal{W}_{I, L}=} & \frac{P T}{\pi^{2} 2^{L} M} \sum_{i=0}^{2^{I}-1} \sin ^{2}\left(\pi z_{i}\right) \sum_{u=1}^{L} 2^{L-u} \\
& \sum_{n=0}^{2^{-I-u} N-1} W_{L, 2^{-I} N}\left(u, n ; 2^{I}+1, z_{i}\right)
\end{aligned}
$$

with $W_{L, \widetilde{N}}(u, n ; \xi, \varrho)$ defined in (35).

\section{Reduced Hadamard PRecoded OFDM}

New orthogonal precoders for ZP-OFDM and BCP-OFDM are presented here based on reduced Hadamard matrix. First, consider the $2^{L} \times 2^{L}$ Hadamard matrix that contains elements \pm 1 and has the $\beta$ th column vector characterized by $\underline{h}_{\beta} \triangleq\left[h_{0, \beta}, h_{1, \beta}, \ldots, h_{2^{L}-1, \beta}\right]^{t}$ with $h_{\alpha, \beta} \triangleq(-1)^{\sum_{l=1}^{L} \alpha_{l} \beta_{l}}$ for $\alpha, \beta \in \mathcal{Z}_{2^{L}}$, where $\alpha=\sum_{l=1}^{L} \alpha_{l} 2^{l-1}$ and $\beta=\sum_{l=1}^{L} \beta_{l} 2^{l-1}$ are binary expansions of $\alpha$ and $\beta$ with $\alpha_{l}, \beta_{l} \in \mathcal{Z}_{2}$. This matrix has two useful properties: 1) All column vectors are orthogonal, i.e., $\underline{h}_{\beta_{1}}^{t} \underline{h}_{\beta_{2}}=2^{L} \delta_{\beta_{1}, \beta_{2}}$. 2) Any $\underline{h}_{\beta}$ with $\beta \neq 0$ consists of equal numbers of elements \pm 1 , i.e., $\underline{h}_{\beta}^{t} \underline{1}=0$ for $\beta \neq 0$ with $\underline{1}$ an all-one vector. Motivated by these two properties, the following spectral precoders $\mathcal{H}_{L}$ and $\mathcal{H}_{I, L}$ are constructed from the reduced Hadamard matrix that consists of $2^{L}-1$ columns $\underline{h}_{1}, \underline{h}_{2}, \ldots, \underline{h}_{2^{L}-1}$. The first property warrants that the resultant precoders are orthogonal, while the second property endows the corresponding precoded OFDM signals with fast decaying spectral sidelobes.

A) $\mathcal{H}_{L}$-coded ZP-OFDM: For $L \in \mathcal{Z}_{\log _{2} N}^{+}$, the $L$ th-order precoder $\mathcal{H}_{L}$ is defined as

$$
\begin{aligned}
G_{n 2^{L}+\alpha, n\left(2^{L}-1\right)+\beta-1} & =2^{-\frac{L}{2}}(-1)^{\alpha} h_{\alpha, \beta} \\
n & \in \mathcal{Z}_{2^{-L} N}, \alpha \in \mathcal{Z}_{2^{L}}, \beta \in \mathcal{Z}_{2^{L}-1}^{+}
\end{aligned}
$$

and $G_{n, m}=0$ otherwise. In the case, $M=N\left(1-2^{-L}\right)$. By Hadamard properties, these $G_{n, m}$ 's are readily shown to satisfy (7) and Constraint $l$ with $K=1$, respectively. Therefore, $\mathcal{H}_{L}$ is orthogonal and $\mathcal{H}_{L}$-coded ZP-OFDM provides small power spectral sidelobes decaying asymptotically as $f^{-4}$. Using (25) in (9) gives

$$
\begin{aligned}
\left.\mathcal{S}_{L P}^{Z P}(f)\right|_{\mathcal{H}_{L}}= & \frac{P T_{d}}{\pi^{2} 2^{L} M} \sin ^{2}(\pi z) \\
& \sum_{n=0}^{2^{-L} N-1} \sum_{\beta=1}^{2^{L}-1} H_{L, N}(n, \beta ; 1, z)
\end{aligned}
$$

with $H_{L, \tilde{N}}(m, \beta ; \xi, \varrho)$ defined in (33). From [11, eq. (24)], the $\mathcal{S}_{L P}^{Z P}(f)$ for $\mathcal{W}_{L}$-coded ZP-OFDM can be alternatively expressed in the form of (9), as

$$
\begin{aligned}
\left.\mathcal{S}_{L P}^{Z P}(f)\right|_{\mathcal{W}_{L}=}= & \frac{P T_{d}}{\pi^{2} 2^{L} M} \sin ^{2}(\pi z) \sum_{u=1}^{L} 2^{L-u} \\
& \sum_{n=0}^{2^{-u} N-1} W_{L, N}(u, n ; 1, z) .
\end{aligned}
$$

Although seemingly different, $\left.\mathcal{S}_{L P}^{Z P}(f)\right|_{\mathcal{H}_{L}}$ and $\left.\mathcal{S}_{L P}^{Z P}(f)\right|_{\mathcal{W}_{L}}$ are shown in Appendix B to be equivalent and thus result in the following proposition.

Proposition 3: $\mathcal{H}_{L}$-coded ZP-OFDM offers the same spectrum as $\mathcal{W}_{L}$-coded ZP-OFDM.

Because the nonzero precoder coefficients for $\mathcal{H}_{L}$ are all real with the same magnitude $2^{-\frac{L}{2}}$ (see (25)), the magnitude can be properly accounted for when calibrating the signal power during system operation and, thus, the encoding and decoding functions in (2) and (8) can be realized without multiplication. This implies that $\mathcal{H}_{L}$-coded ZP-OFDM is simpler to realize than $\mathcal{W}_{L}$-coded ZP-OFDM, while both providing the same spectral performance.

B) $\mathcal{H}_{I, L}$-coded BCP-OFDM: For $L \in \mathcal{Z}_{\log _{2} N-I}^{+}$and $I \in$ $\mathcal{Z}_{\log _{2} N-1}^{+}$, the $L$ th-order precoder $\mathcal{H}_{I, L}$ for BCP-OFDM with $T_{g}=2^{-I} T_{d}$ is defined as

$$
\begin{gathered}
g_{n 2^{L}+\alpha, n\left(2^{L}-1\right)+\beta-1}=2^{-\frac{L}{2}} h_{\alpha, \beta} \\
n \in \mathcal{Z}_{2^{-I-L} N}, \alpha \in \mathcal{Z}_{2^{L}}, \beta \in \mathcal{Z}_{2^{L}-1}^{+}
\end{gathered}
$$

and $g_{n, m}=0$ otherwise. In the case, $M=N\left(1-2^{-L}\right)$. Due to Hadamard properties, these $g_{n, m} \mathrm{~s}$ satisfy Constraint $2 c$ with $K=1$ and (17). Thus, $\mathcal{H}_{I, L}$ is orthogonal and $\mathcal{H}_{I, L}$-coded BCP-OFDM with $T_{g}=2^{-I} T_{d}$ provides small power spectral sidelobes decaying asymptotically as $f^{-4}$. Using (28) in (15) gives

$$
\begin{aligned}
\left.\mathcal{S}_{L P}^{B C P}(f)\right|_{\mathcal{H}_{I, L}=} & \frac{P T}{\pi^{2} 2^{L} M} \sum_{i=0}^{2^{I}-1} \sin ^{2}\left(\pi z_{i}\right) \sum_{n=0}^{2^{-L-I} N-1} \\
& \sum_{\beta=1}^{2^{L}-1} H_{L, 2^{-I} N}\left(n, \beta ; 2^{I}+1, z_{i}\right) .
\end{aligned}
$$

From [11, eq. (26)], the $\mathcal{S}_{L P}^{C P}(f)$ for $\mathcal{V}_{\log _{2} N-I^{-c o d e d} \mathrm{CP} \text { - }}$ OFDM with $T_{g}=2^{-I} T_{d}$ can be alternatively expressed in the form of (11), as

$$
\begin{aligned}
\left.\mathcal{S}_{L P}^{C P}(f)\right|_{\mathcal{V}_{\log _{2} N-I}=}= & \frac{P T}{\pi^{2} M} \sum_{i=0}^{2^{I}-1} \sin ^{2}\left(\pi z_{i}\right) \sum_{u=1}^{\log _{2} N-I} 2^{-u} \\
& \sum_{n=0}^{2^{-I-u} N-1} V_{\log _{2} N-I}\left(u, n ; 2^{I}+1, z_{i}\right)
\end{aligned}
$$

with $V_{\widetilde{L}}(u, n ; \xi, \varrho)$ defined in (34). In Appendix B, $\left.\mathcal{S}_{L P}^{B C P}(f)\right|_{\mathcal{H}_{I, L}}$ and $\left.\mathcal{S}_{L P}^{B C P}(f)\right|_{\mathcal{W}_{I, L}}$ are proven to be identical and equal to $\left.\mathcal{S}_{L P}^{C P}(f)\right|_{\mathcal{V}_{\log _{2} N-I}}$ when $L=\log _{2} N-I$. This yields 


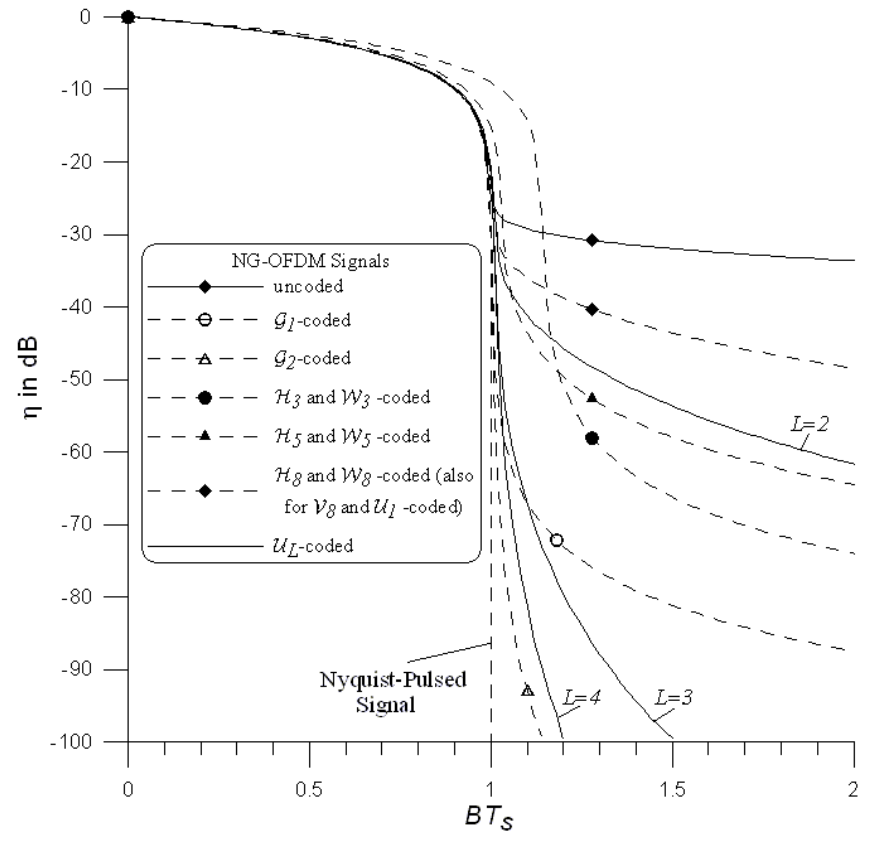

Fig. 2. Fractional out-of-band power characteristics of $\mathcal{U}_{L}$-coded NG-OFDM signals with $N=256$.

Proposition 4: For $T_{g}=2^{-I} T_{d}$ with $I \in \mathcal{Z}_{\log _{2} N-1}^{+}, \mathcal{H}_{I, L^{-}}$ coded BCP-OFDM offers the same spectrum as $\mathcal{W}_{I, L}$-coded BCP-OFDM. Moreover, both $\mathcal{H}_{I, \log _{2} N-I}$ and $\mathcal{W}_{I, \log _{2} N-I}$ -coded BCP-OFDM offer the same spectrum as $\mathcal{V}_{\log _{2} N-I^{-}}$ coded CP-OFDM.

Like $\mathcal{H}_{L}$, the encoding and decoding functions in (13) and (18) for $\mathcal{H}_{I, L}$ can be realized without multiplication because its nonzero precoder coefficients have the same magnitude $2^{-\frac{L}{2}}$. This implies that $\mathcal{H}_{I, L}$-coded BCP-OFDM is simpler to realize than $\mathcal{W}_{I, L}$-coded BCP-OFDM and $\mathcal{V}_{\log _{2} N-I \text {-coded }}$ CP-OFDM.

\section{Power Spectral Performance Characteristics}

In the following, power spectral compactness is characterized by the decibel out-of-band power fraction $\eta=$ $10 \log _{10}\left(1-\frac{1}{P} \int_{-B / 2}^{B / 2} \mathcal{S}_{L P}(f) d f\right)$, which denotes the fraction of total power that is not captured within the frequency band $[-B / 2, B / 2]$. Specifically, the results on $\eta$ are presented with respect to the normalized bandwidth $B T_{s}$ so that the spectral efficiency can be compared among different signals with the same data symbol rate. Here, spectral efficiency is referred to as the reciprocal of $B T_{s}$ required to achieve a given $\eta$. In order to achieve the same $\eta$, the signal requiring a smaller $B T_{s}$ can exhibit higher spectral efficiency.

When $T_{g}=0$, the analysis on ZP-OFDM can also be applied to OFDM without guard intervals (NG-OFDM). Thus, $\mathcal{S}_{L P}^{N G}(f)$ for NG-OFDM has the same form as $\mathcal{S}_{L P}^{Z P}(f)$ with $T_{d}$ replaced by $T$ and the spectral efficiency for NG-OFDM can be directly transformed to ZP-OFDM by scaling down with a factor $T_{d} / T$ [11]. Fig. 2 illustrates the spectral efficiency of $\mathcal{U}_{L}$-coded NG-OFDM. Also compared therein are uncoded and other spectrally precoded NG-OFDMs, and the ideal singlecarrier Nyquist-pulsed signal. It is numerically found that $\mathcal{U}_{1}, \mathcal{H}_{8}, \mathcal{W}_{8}$, and $\mathcal{V}_{8}$-coded NG-OFDMs perform the same.

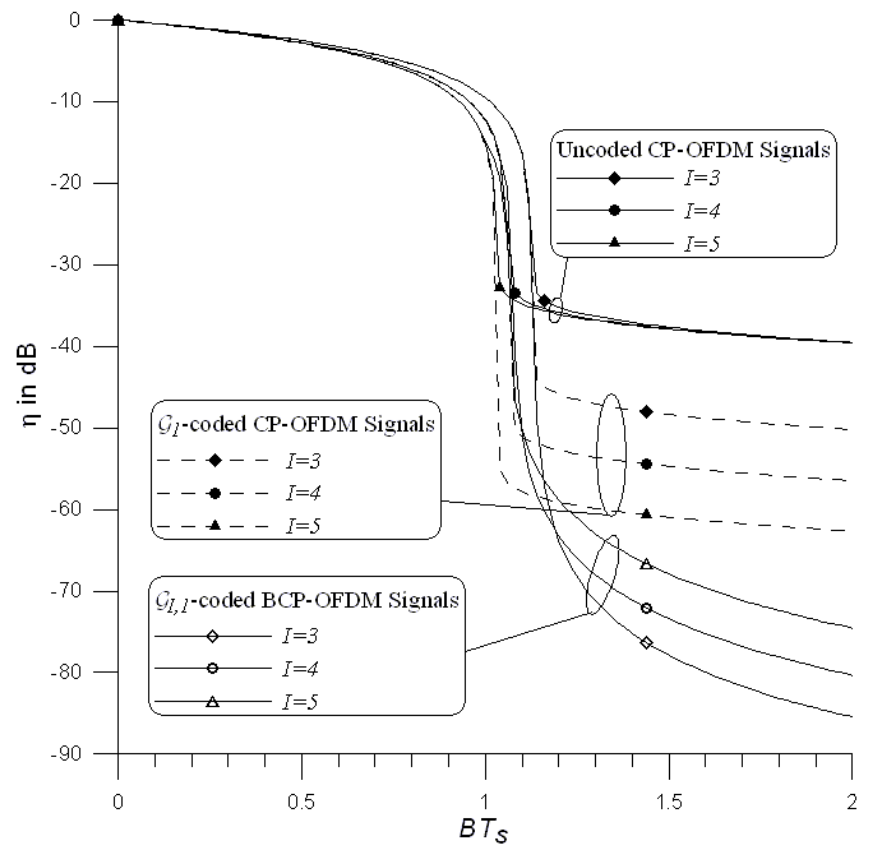

Fig. 3. Fractional out-of-band power characteristics of $\mathcal{G}_{I, 1}$-coded BCPOFDM signals with $N=1024$ and $T_{g}=2^{-I} T_{d}$.

As shown, $\mathcal{U}_{L}$-coded NG-OFDM provides higher spectral efficiency for a larger $L$. Particularly, $\mathcal{U}_{L}$-coded NG-OFDM with a medium $L$ can significantly outperforms uncoded and $\mathcal{H}_{L^{\prime}}, \mathcal{W}_{L^{\prime}}$, and $\mathcal{V}_{L^{\prime}}$-coded NG-OFDM, and perform very close to Nyquist-pulsed signal and $\mathcal{G}_{L^{\prime \prime}}$-coded NG-OFDM.

Fig. 3 illustrates the spectral efficiency of $\mathcal{G}_{I, 1}$-coded BCPOFDM with $T_{g}=2^{-I} T_{d}$. Uncoded and $\mathcal{G}_{1}$-coded CPOFDMs are also compared. Several performance trends can be observed: First, when $B T_{s}$ is large (say, beyond 1.2) and increased, $\eta$ decreases more remarkably for $\mathcal{G}_{I, 1}$-coded BCPOFDM (which decays asymptotically as $f^{-4}$ ) than for uncoded and $\mathcal{G}_{1}$-coded CP-OFDMs (which decay asymptotically as $f^{-2}$ ). The performance prevalence is, however, lost in the $B T_{s}$ range enclosing $(1,1.2)$ because $\mathcal{G}_{I, 1}$-coded $\mathrm{BCP}$ OFDM has a smaller precoding rate $\lambda$ (i.e., a shorter $T$ ) than uncoded and $\mathcal{G}_{1}$-coded CP-OFDMs. Second, when $B T_{s}$ is large enough, $\mathcal{G}_{I, 1}$-coded BCP-OFDM provides smaller $\eta$ as the guard ratio is larger, a trend on the contrary to $\mathcal{G}_{1}$ coded CP-OFDMs. This implies from (13) that precoding spectrally near subcarriers prevails over precoding spectrally far subcarriers for $\mathcal{G}_{I, 1}$-coded BCP-OFDM. As observed by the author, a similar trend also holds for orthogonally precoded BCP-OFDMs. Third, $\mathcal{G}_{I, 1}$-coded BCP-OFDM provides higher spectral efficiency than uncoded and $\mathcal{G}_{1}$-coded CP-OFDMs when the required $\eta$ is small (say, $-70 \mathrm{~dB}$ ), especially when $I$ is small. Although not presented explicitly, the above performance trends also apply to $\mathcal{G}_{I, L}$-coded BCP-OFDM with other larger $L$.

The spectral efficiencies of orthogonally and correlatively precoded BCP-OFDMs with $T_{g} / T_{d}=2^{-3}$ are compared in Fig. 4. Recall that $\mathcal{H}_{I, L}$ and $\mathcal{W}_{I, L}$-coded BCP-OFDMs have the same spectrum and are spectrally equivalent to $\mathcal{V}_{\log _{2} N-I^{-}}$ coded CP-OFDM when $L=\log _{2} N-I$. As shown, all BCPOFDMs provide higher spectral efficiency than uncoded CP- 


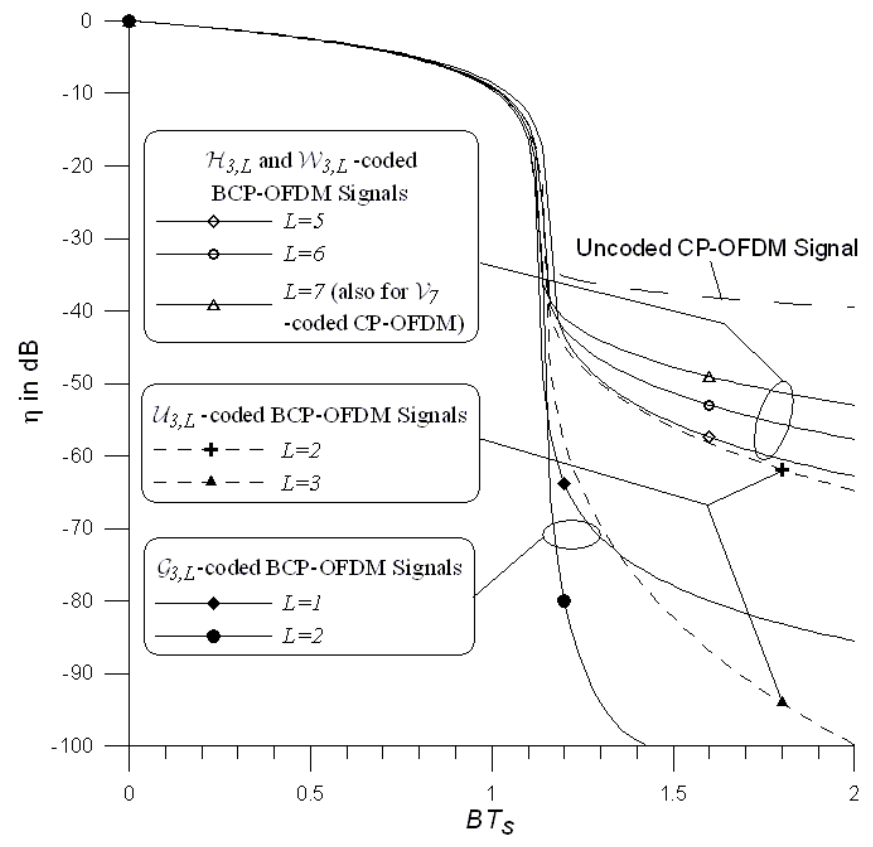

Fig. 4. Fractional out-of-band power characteristics of $\mathcal{H}_{3, L}, \mathcal{U}_{3, L}, \mathcal{W}_{3, L}$, and $\mathcal{G}_{3, L}$-coded BCP-OFDM signals with $N=1024$ and $T_{g}=2^{-3} T_{d}$.

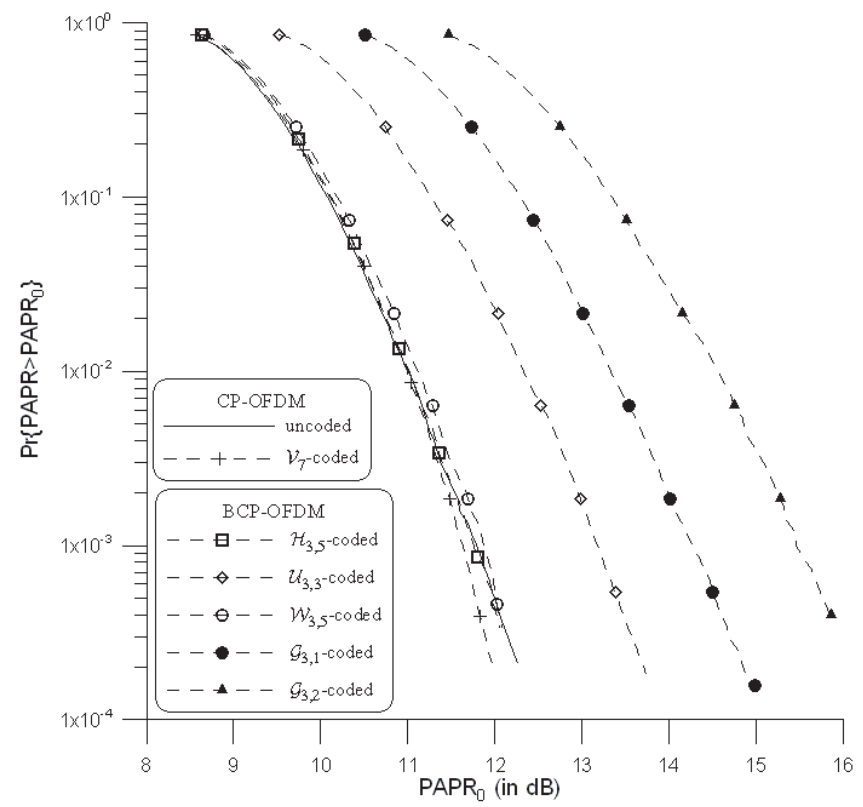

Fig. 5. CCDF of PAPR for BCP-OFDM and CP-OFDM schemes with $N=1024, T_{g}=2^{-3} T_{d}$, and QPSK component modulation.

OFDM when the required $\eta$ is small (say, $-40 \mathrm{~dB}$ ). When $B T_{s}$ is large enough, $\mathcal{H}_{3, L}$ and $\mathcal{W}_{3, L}$-coded BCP-OFDMs with $L<\log _{2} N-I$ provide smaller $\eta$ than $\mathcal{V}_{\log _{2} N-I \text {-coded }}$ CP-OFDM. When the required $\eta$ is very small (say, $-70 \mathrm{~dB}$ ), $\mathcal{G}_{3, L}$ and $\mathcal{U}_{3,3}$-coded BCP-OFDMs can provide much higher spectral efficiency than all $\mathcal{H}_{3, L^{\prime}}$ and $\mathcal{W}_{3, L^{\prime}}$-coded BCPOFDMs.

A low peak-to-average power ratio (PAPR) at the transmitter output is desirable for most OFDM applications [24]. Because data block constellation is altered before OFDM modulation, spectral precoding varies the distribution of PAPR. Such an effect is illustrated in Fig. 5. The transmitted OFDM signals using QPSK component modulations are oversampled by a factor of 4 and $10^{6}$ random OFDM blocks are generated to obtain the complementary cumulative distribution functions (CCDFs) of PAPR. As indicated, the PAPR characteristic of OFDM is not substantially altered for $\mathcal{H}, \mathcal{V}$ and $\mathcal{W}$ types of spectral precoding, but is remarkably traded off for highly spectrally efficient $\mathcal{G}$ and $\mathcal{U}$ types of spectral precoding.

\section{ERRor PERFORMANCE AND COMPLEXITY CHARACTERISTICS}

A) Spectral Decoding: When the channel response is available and perfect alignment in timing and frequency is achieved at the receiver, spectrally precoded OFDM signals can be coherently demodulated. The standard FFT-based OFDM receiver is as illustrated in Fig. 1. The received waveform is first manipulated by the guard process to extract useful signals. Specifically, the guard process removes guard signals for BCPOFDM and CP-OFDM, and overlaps and adds the guard signals to the received signals in data block intervals for ZPOFDM [25]. Following the guard process, the FFT is applied over each data block interval to yield $\left\{R_{n} ; n \in \mathcal{Z}_{N}\right\}$ with $R_{n}$ representing the received complex symbol on the $n$th subcarrier. The spectral decoder is then used to recover $\left\{D_{m} ; m \in\right.$ $\left.\mathcal{Z}_{M}\right\}$ by processing $\left\{R_{n} ; n \in \mathcal{Z}_{N}\right\}$. When the guard interval length is long enough to reject the intersymbol interference (ISI), $R_{n}$ can be analytically modeled by $R_{n}=H_{n} B_{n}+Y_{n}$ where $H_{n}$ denotes the known complex channel response on the $n$th subcarrier and $Y_{n} \mathrm{~s}$ are identically-distributed circularlysymmetric complex AWGN samples with zero mean and variance $\sigma_{Y}^{2}$. For CP-OFDM and BCP-OFDM, $Y_{n} \mathrm{~s}$ are mutually independent. For ZP-OFDM, $Y_{n} \mathrm{~s}$ are dependent and have correlations $E\left\{Y_{n} Y_{m}^{*}\right\}=\sigma_{Y}^{2} \widetilde{\rho}_{n-m}$ for $n \neq m$ where $\widetilde{\rho}_{k} \triangleq \frac{1}{N+J} \sum_{l=0}^{J-1} \exp \left\{-j \frac{2 \pi}{N} l k\right\}$ with $J$ being the number of $\mathrm{ZP}$ symbols in each guard interval.

When the precoder is correlative, the decision on $\left\{D_{m} ; m \in\right.$ $\left.\mathcal{Z}_{M}\right\}$ has to be made blockwise because block data are correlatively combined. In the case, the maximum-likelihood (ML) block decoding can be applied to achieve the minimum probability of error in block decision and efficiently realized by Viterbi algorithm for CP-OFDM [26]. The Viterbi block decoding of $\mathcal{G}_{L}$-coded OFDM has been dealt with in [10]. ${ }^{7}$ By first partitioning $\left\{R_{n} ; n \in \mathcal{Z}_{N}\right\}$ into subblocks $\left\{R_{i+n 2^{I}} ; n \in\right.$ $\left.\mathcal{Z}_{2^{-I} N}\right\}$ for $i \in \mathcal{Z}_{2^{I}}$, and then applying Viterbi block decoding on each subblock, the same ML decoding rule as in [10] can be used for $\mathcal{G}_{I, L}$-coded BCP-OFDM.

When the precoder is orthogonal, the decision on $\left\{D_{m} ; m \in \mathcal{Z}_{M}\right\}$ may be done blockwise or symbolwise. Block decoding can be designed based on the same ML principle as in the short block spreading schemes in [27]. In general, the ML block decoding approach can exploit the advantage of data spreading over subcarriers and achieve diversity gain on error performance in DS or multipath fading channels [27]. However, such block decoding approaches

${ }^{7}$ The Viterbi block decoding algorithm in [10] is an ML algorithm for $\mathcal{G}_{L^{-}}$ coded CP-OFDM, but not for $\mathcal{G}_{L}$-coded ZP-OFDM because noise correlations among $Y_{n} \mathrm{~s}$ are not manipulated in the latter case. However, when the signalto-noise power ratio is large or the guard ratio $J / N$ is small, the Viterbi block decoding algorithm for $\mathcal{G}_{L}$-coded ZP-OFDM approaches asymptotically to an $\mathrm{ML}$ one in that the effect of noise correlation tends to be insignificant. 
are complicated to realize for the proposed orthogonally precoded OFDMs with large block sizes and not pursued in the paper. Instead, the zero-forcing (ZF) approach [11] that recovers $\left\{D_{m} ; m \in \mathcal{Z}_{M}\right\}$ symbolwise is simpler to implement and considered here. Specifically, the $\mathrm{ZF}$ decoding rule is to find the candidate data symbol $\widehat{D}_{m}$ that is closest in Euclidean distance to the metric $X_{m}$ for each $m \in \mathcal{Z}_{M}$, where $X_{m} \triangleq \sum_{n=0}^{N-1} G_{n, m}^{*} R_{n} / H_{n}$. Using (8), $X_{m}$ can be equivalently modeled as $X_{m}=D_{m}+U_{m}$ where $U_{m} \triangleq \sum_{n=0}^{N-1} G_{n, m}^{*} Y_{n} / H_{n}$ is circularly symmetric complex Gaussian with zero mean. This is exactly the same as the ZF subcarrier demodulation model for uncoded OFDM [1]-[3]. Thus, when the same component modulation is used and $\left|H_{n}\right| \mathrm{s}$ are deterministic (e.g., AWGN and DS channels), the bit error rates of $k$ th data symbol demodulation for uncoded and orthogonally precoded OFDMs have the same function $P_{b}(\gamma(k))$ which is inversely proportional to the signal-to-noise power ratio (SNR) $\gamma(k)$ with $\gamma(k)$ given by $\gamma_{u n c}(k)$ and $\gamma_{c o d}(k)$, respectively. Here, $\gamma_{u n c}(k)$ for $k \in \mathcal{Z}_{N}$ and $\gamma_{c o d}(k)$ for $k \in \mathcal{Z}_{M}$ are the SNRs on the $k$ th subcarrier for uncoded OFDM and on subchannel $k$ for orthogonally precoded OFDM, respectively, and are given by $\gamma_{u n c}(k) \triangleq \sigma_{Y}^{-2}\left|H_{k}\right|^{2}$ for all OFDM formats, $\gamma_{c o d}(k) \triangleq \sigma_{Y}^{-2}\left(\sum_{n=0}^{N-1}\left|G_{n, k}\right|^{2} /\left|H_{n}\right|^{2}\right)^{-1}$ for CP-OFDM and BCP-OFDM, and $\gamma_{\text {cod }}(k) \triangleq$ $\sigma_{Y}^{-2}\left(\sum_{n=0}^{N-1} \sum_{m=0}^{N-1} G_{n, k}^{*} G_{m, k} \widetilde{\rho}_{n-m}\left(H_{n} H_{m}^{*}\right)^{-1}\right)^{-1}$ for ZPOFDM. In the case, these ZF schemes can be characterized by the average bit error rate (ABER) $w \sum_{k} P_{b}(\gamma(k))$ with $w=N^{-1}$ and $M^{-1}$ for uncoded and spectrally precoded schemes, respectively. If $\left|H_{n}\right| \mathrm{s}$ are random but remain unchanged over one block (e.g., time-nonselective flat or multipath fading channels), the ABER can be obtained by simulating the channel realizations for all $\left|H_{n}\right| \mathrm{s}$ and averaging the computed values of $w \sum_{k} P_{b}(\gamma(k))$ over these realizations. Particularly, when $\left|H_{n}\right| \mathrm{s}$ are constant (e.g., AWGN or flat fading channels), $\gamma_{u n c}(n)$ and $\gamma_{c o d}(m)$ are equal for CP-OFDM and BCP-OFDM. Therefore, all orthogonally precoded CP-OFDMs and BCP-OFDMs perform the same as uncoded CP-OFDM on AWGN and flat fading channels.

B) ABER Results: Here, we study the ABER characteristics among uncoded and $\mathcal{V}_{L}$-coded CP-OFDM schemes and various spectrally precoded BCP-OFDM schemes with Graylabeled 16-ary QAM component modulations on AWGN and two-ray fading channels. $E_{b} / N_{0}$ is defined as the bit SNR for useful data blocks. The two-ray fading channel is modeled by $H_{l}=\left.H(z)\right|_{z=\exp \{j 2 l \pi / N\}}$ where $H(z)$ is defined by $H(z)=p+q z^{-\tau}$ with $p$ and $q$ denoting the complex channel gains corresponding to the first path and the delayed path on integer delay $\tau$. Here, $p$ and $q$ are modeled to be independent circularly symmetric complex Gaussian noise samples with means $E\{p\}=[\Omega /(1+\Omega)]^{1 / 2}$ and $E\{q\}=0$ and variances $\operatorname{Var}\{p\}=E\left\{|p-E\{p\}|^{2}\right\}=(1+\Omega)^{-1}\left(1+\Lambda^{-1}\right)^{-1}$ and $\operatorname{Var}\{q\}=E\left\{|q|^{2}\right\}=(1+\Omega)^{-1}(1+\Lambda)^{-1}$. By modeling so, $p$ and $q$ represent Rician and Rayleigh paths, respectively, where $\Omega$ denotes the ratio of the direct power (i.e., $|E\{p\}|^{2}$ ) to the sum of diffuse powers (i.e., $\operatorname{Var}\{p\}+\operatorname{Var}\{q\}$ ) and $\Lambda$ is the ratio of diffuse powers given by $\Lambda=\operatorname{Var}\{p\} / \operatorname{Var}\{q\}$. Note
TABLE II

AVERAGE BIT ERROR RATE CHARACTERISTICS OF GRAY-LABELED BCP-OFDM AND CP-OFDM WITH $N=1024,16$-ARY QAM COMPONENT MODULATION, $T_{g}=2^{-3} T_{d}$, AND $E_{b} / N_{0}=15 \mathrm{DB}$ ON VARIOUS TWO-RAY FADING CHANNELS WITH $\Omega=3$ AND $\Lambda=1$. FOR EACH ALGORITHM, $A B E R_{\max }$ AND $A B E R_{\min }$ DENOTE THE MAXIMUM AND MINIMUM ABER VALUES CORRESPONDING TO $\tau_{\max }$ AND $\tau_{\min }$, RESPECTIVELY, WHICH ARE OBTAINED BY SEARCHING ALL ABER VALUES FOR $\tau \in \mathcal{Z}_{128}^{+}$.

\begin{tabular}{c||c|c}
\hline Algorithm & $\left(A B E R_{\max }, \tau_{\max }\right)$ & $\left(A B E R_{\min }, \tau_{\min }\right)$ \\
\hline \hline $\begin{array}{l}\mathcal{G}_{3,1} \text {-coded } \\
\text { BCP-OFDM }\end{array}$ & $\left(3.38 \times 10^{-3}, 61\right)$ & $\left(3.03 \times 10^{-3}, 98\right)$ \\
\hline $\begin{array}{l}\mathcal{G}_{3,2} \text {-coded } \\
\text { BCP-OFDM }\end{array}$ & $\left(3.69 \times 10^{-3}, 127\right)$ & $\left(3.25 \times 10^{-3}, 16\right)$ \\
\hline \hline $\begin{array}{l}\mathcal{H}_{3,5} \text {-coded } \\
\text { BCP-OFDM }\end{array}$ & $\left(1.37 \times 10^{-3}, 50\right)$ & $\left(6.56 \times 10^{-4}, 16\right)$ \\
\hline $\begin{array}{l}\mathcal{H}_{3,6} \text {-coded } \\
\text { BCP-OFDM }\end{array}$ & $\left(1.44 \times 10^{-3}, 50\right)$ & $\left(7.16 \times 10^{-4}, 110\right)$ \\
\hline $\begin{array}{l}\mathcal{H}_{3,7} \text {-coded } \\
\text { BCP-OFDM }\end{array}$ & $\left(1.44 \times 10^{-3}, 78\right)$ & $\left(7.08 \times 10^{-4}, 48\right)$ \\
\hline \hline $\begin{array}{l}\mathcal{U}_{3,3} \text {-coded } \\
\text { BCP-OFDM }\end{array}$ & $\left(1.12 \times 10^{-3}, 5\right)$ & $\left(6.69 \times 10^{-4}, 34\right)$ \\
\hline \hline $\begin{array}{l}\mathcal{V}_{7} \text {-coded } \\
\text { CP-OFDM }\end{array}$ & $\left(1.05 \times 10^{-3}, 50\right)$ & $\left(6.83 \times 10^{-4}, 101\right)$ \\
\hline \hline $\mathcal{W}_{3,5}$-coded \\
BCP-OFDM & $\left(1.12 \times 10^{-3}, 5\right)$ & $\left(6.70 \times 10^{-4}, 34\right)$ \\
\hline $\begin{array}{l}\mathcal{W}_{3,6} \text {-coded } \\
\text { BCP-OFDM }\end{array}$ & $\left(1.13 \times 10^{-3}, 5\right)$ & $\left(6.71 \times 10^{-4}, 34\right)$ \\
\hline $\begin{array}{l}\mathcal{W}_{3,7} \text {-coded } \\
\text { BCP-OFDM }\end{array}$ & $\left(1.13 \times 10^{-3}, 5\right)$ & $\left(6.72 \times 10^{-4}, 34\right)$ \\
\hline \hline $\begin{array}{l}\text { Uncoded } \\
\text { OFDM }\end{array}$ & $\left(1.04 \times 10^{-3}, 122\right)$ & $\left(7.82 \times 10^{-4}, 110\right)$ \\
\hline
\end{tabular}

that the sum of direct and diffuse powers is normalized to one. The results of uncoded and orthogonally precoded ZF schemes are thus obtained by averaging $w \sum_{k} P_{b}(\gamma(k))$ over simulated channel realizations for $p$ and $q$ with $P_{b}(\gamma(k))$ given by [26, eq. 10.36a], while those of correlatively precoded ML block schemes are obtained by simulating the ML block decoding.

As illustrated in Table II, the ABER characteristics of uncoded and spectrally precoded schemes on two-ray fading channels depend on, but do not vary significantly with, the delay spread $\tau$. In comparison with uncoded and orthogonally precoded schemes, correlatively precoded schemes are relatively insensitive to $\tau$.

Figs. 6 and 7 give the ABER comparison among uncoded and spectrally precoded schemes on AWGN and various two-ray fading channels. The two-ray fading channels are characterized by fixing $\tau=108$ and $\Lambda=1$ and varying $\Omega$. The smaller the $\Omega$, the heavier the two-ray channel fades. As shown, orthogonally precoded ZF schemes perform the same on AWGN and comparably on all two-ray channels as uncoded ZF scheme. When the two-ray channel fades lightly (e.g., $\Lambda=7$ ), uncoded and orthogonally precoded ZF schemes prevail over correlatively precoded ML block schemes significantly. However, such performance prevalence is reversed for low ABER regions when the two-ray channel fades heavier (e.g., $\Lambda=3$ and 0 ). Such performance trends occur because the uncoded and orthogonally precoded ZF schemes suffer noise enhancement when subchannels are in deep fade but the correlatively precoded block schemes can exploit the advantage of data correlation over distant subcarriers and thus exhibit diversity gain in heavily faded channels. 


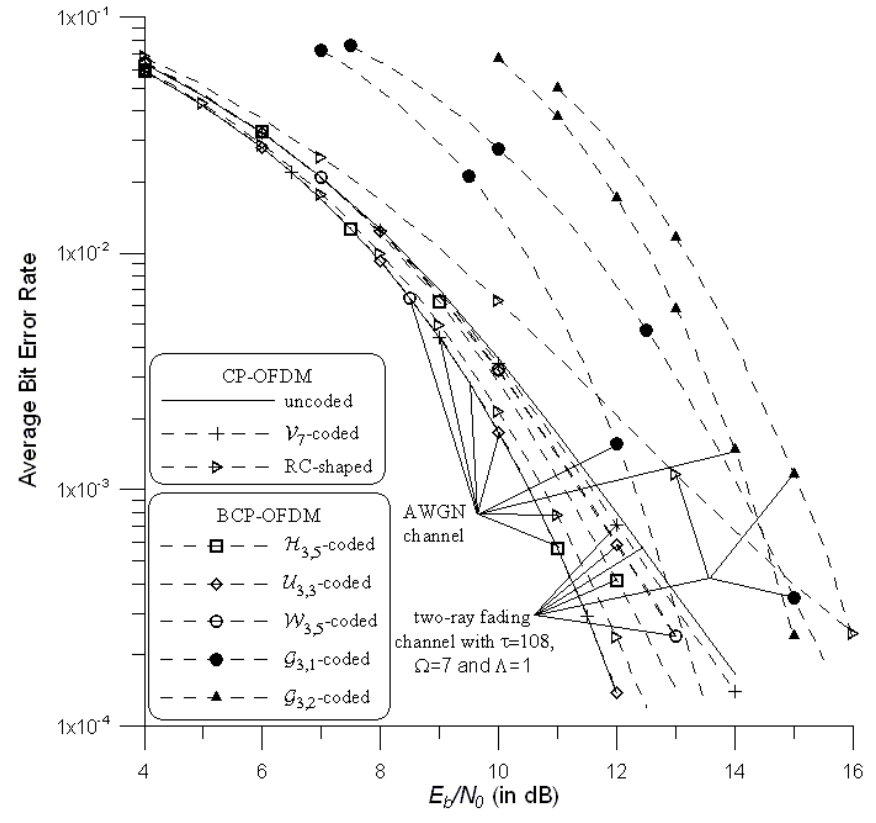

Fig. 6. Average bit error rate characteristics of Gray-labeled BCP-OFDM and CP-OFDM with $N=1024, T_{g}=2^{-3} T_{d}$, and 16-ary QAM component modulation on AWGN and two-ray fading channels.

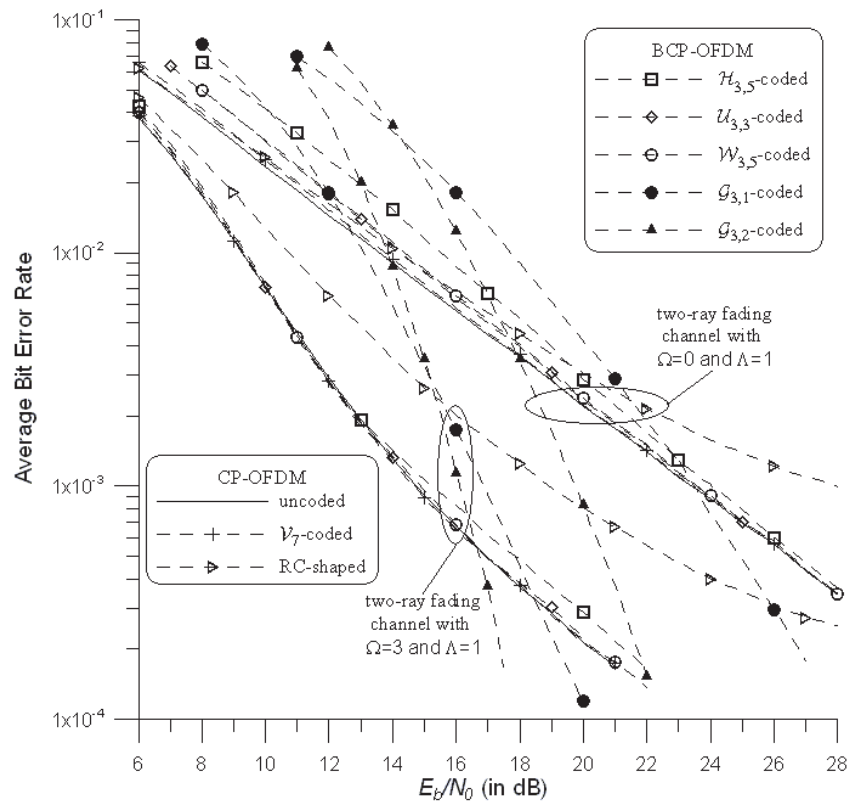

Fig. 7. Average bit error rate characteristics of Gray-labeled BCP-OFDM and CP-OFDM with $N=1024, T_{g}=2^{-3} T_{d}$, and 16-ary QAM component modulation on two-ray fading channels with $\tau=108$.

In practical OFDM transmitter, frontend filtering [17] or waveform shaping [14] is commonly applied after inverse FFT and guard insertion to reduce sidelobe powers. Such processes generally cause ISI or intercarrier interference (ICI) at the receiver due to transmitted waveform distortion. The effect is also illustrated in Figs. 6 and 7. The transmitted waveform after frontend filtering is modeled as

$$
s_{R C}(t)=\rho \operatorname{Re}\left\{\sum_{n=0}^{N-1} B_{n} \exp \left\{j\left(\omega_{0}+n \omega_{d}\right) t\right\}\right\} p_{R C}(t)
$$

on the nominal block duration $-T_{g}-T_{d} / 2 \leq t \leq T_{d} / 2$. Here,
$p_{R C}(t)$ is a (unit-energy) time-domain raised cosine (RC) pulse defined by $p_{R C}(t)=\widetilde{\alpha} \cdot R C\left((1+\widetilde{\beta})\left(t+T_{g} / 2\right) / T, \widetilde{\beta}\right)$ where $\widetilde{\beta}$ is the time-domain rolloff factor, $\widetilde{\alpha}=[T(1-$ $\widetilde{\beta} / 4) /(1+\widetilde{\beta})]^{-1 / 2}$, and $R C(x, y)$ is defined by $R C(x, y)=1$ if $|x| \leq(1-y) / 2, R C(x, y)=\cos ^{2}(\pi(2|x|-1+y) /(4 y))$ if $(1-y) / 2<|x| \leq(1+y) / 2$, and $R C(x, y)=0$ otherwise. Note that the RC pulse is a shaping of rectangular pulse only at pulse edges on $\frac{1-\widetilde{\beta}}{1+\tilde{\beta}} \frac{T}{2}<\left|t+\frac{T_{g}}{2}\right| \leq \frac{T}{2}$ and does not cause ISI. The standard FFT-based ZF CP-OFDM receiver is used to detect $s_{R C}(t)$. Because $s_{R C}(t)$ is not matched, the FFTbased receiver suffers ICI. In the simulation, $\widetilde{\beta}=0.0041$ and this rolloff factor results in only $0.817 \%$ of shaping on each edge of rectangular pulse. By this setup, the RC-shaped CP-OFDM provides $\eta=-51.08 \mathrm{~dB}$ at $B T_{s}=1.34$, which is also achieved by $\mathcal{H}_{3,5}$ and $\mathcal{W}_{3,5}$-coded BCP-OFDM (see Fig. 4). Note that $\mathcal{U}_{3,3}, \mathcal{G}_{3,1}$ and $\mathcal{G}_{3,2}$-coded BCP-OFDM can provide $\eta=-72.71,-73.02$ and $-96.77 \mathrm{~dB}$, respectively, at $B T_{s}=1.34$, and thus are more spectrally efficient. For high SNRs, the $R C$-shaped scheme is shown in Figs. 6 and 7 to degrade remarkably in error performance from uncoded and spectrally-precoded schemes in AWGN and two-ray fading channels due to ICI.

C) Complexity Comparison: Spectrally precoded OFDMs differ from uncoded OFDM in the use of spectral encoder and decoder, and thus require additional complexity to realize. In Table III, the additional complexities required for digitally realizing various algorithms are compared. Regarding encoding algorithms, $\mathcal{U}_{L}$ and $\mathcal{U}_{I, L}$ algorithms require the highest complexity which grows with $N^{2}, \mathcal{H}_{L}$ and $\mathcal{H}_{I, L}$ algorithms are the simplest to realize, and the complexities required by the other algorithms are modest and grow linearly with $N L$. As to decoding algorithms, $\mathcal{H}_{L}$ and $\mathcal{H}_{I, L} \mathrm{ZF}$ algorithms still require the least complexity which is comparable with uncoded ZF algorithm. Moreover, $\mathcal{V}_{L}, \mathcal{W}_{L}$ and $\mathcal{W}_{I, L} \mathrm{ZF}$ algorithms require slightly higher complexity than uncoded $\mathrm{ZF}$ algorithm and much less complexity than $\mathcal{G}_{L}$ and $\mathcal{G}_{I, L}$ block algorithms as well as $\mathcal{U}_{L}$ and $\mathcal{U}_{I, L} \mathrm{ZF}$ algorithms.

\section{CONCLUSION}

In the paper, general constraints on spectral precoding are developed for rectangularly pulsed ZP-OFDM and CP-OFDM to guarantee fast decaying power spectral sidelobes that roll off with $f^{-2 K-2}$. According to the constraints, the BCPOFDM signaling format is proposed to facilitate the precoder design of spectrally precoded CP-OFDM with guard ratio specified by $T_{g} / T_{d}=2^{-I}$. Several new precoders including $\mathcal{G}_{I, L}, \mathcal{H}_{I, L}, \mathcal{U}_{I, L}$ and $\mathcal{W}_{I, L}$ are devised for BCP-OFDM to provide fast decaying sidelobes. Besides, new precoders $\mathcal{H}_{L}$ and $\mathcal{U}_{L}$ are also devised to endow ZP-OFDM with fast sidelobe decaying. Particularly, $\mathcal{G}_{I, L}$ and $\mathcal{U}_{I, L}$-coded BCPOFDMs and $\mathcal{U}_{L}$-coded ZP-OFDM can provide extremely small sidelobes decaying asymptotically with $f^{-2 L-2}$, while $\mathcal{H}_{I, L}$ and $\mathcal{W}_{I, L}$-coded BCP-OFDMs and $\mathcal{H}_{L}$-coded ZPOFDM have sidelobes decaying asymptotically with $f^{-4}$. These new schemes are analytically shown to provide more choices of spectral efficiency and realization complexity than previously reported $\mathcal{G}_{L}, \mathcal{V}_{L}$ and $\mathcal{W}_{L}$-coded schemes. 
TABLE III

COMPLEXITY COMPARISON AMONG VARIOUS ENCODING AND DECODING ALGORITHMS. THE ALGORITHMS FOR $\mathcal{G}_{L}, \mathcal{H}_{L}, \mathcal{V}_{L}$, AND $\mathcal{W}_{L}$ PRECODERS CAN BE APPLIED TO ZP-OFDM AND CP-OFDM WITH ARBITRARY $T_{g} / T_{d}$ RATIOS. THE ALGORITHMS FOR $\mathcal{G}_{I, L}, \mathcal{H}_{I, L}$, AND $\mathcal{W}_{I, L}$ ARE ONLY USEFUL FOR BCP-OFDMS WITH $T_{g}=2^{-I} T_{d}$.

\begin{tabular}{|c|c|c|}
\hline Algorithm & $\begin{array}{c}\text { Number of Real } \\
\text { Multiplications } / T\end{array}$ & $\begin{array}{c}\text { Number of Real } \\
\text { Additions } / T \\
\end{array}$ \\
\hline $\begin{array}{c}\mathcal{G}_{L} \text { and } \mathcal{G}_{I, L} \\
\text { Encoding }\end{array}$ & $O(2 N(L+1+2 \varphi))$ & $O(2 N(L+\varphi))$ \\
\hline $\begin{array}{c}\mathcal{H}_{L} \text { and } \mathcal{H}_{I, L} \\
\text { Encoding }\end{array}$ & 0 & $O\left(2 N\left(2^{L}-2\right)\right)$ \\
\hline $\begin{array}{c}\mathcal{U}_{L} \text { and } \mathcal{U}_{I, L} \\
\text { Encoding }\end{array}$ & $O\left(2^{-I} N^{2}+N\right)$ & $O\left(2^{-I} N^{2}-N\right)$ \\
\hline $\begin{array}{c}\mathcal{V}_{L}, \mathcal{W}_{L}, \text { and } \mathcal{W}_{I, L} \\
\text { Encoding }\end{array}$ & $O(2 N L)$ & $O(2 N(L-1))$ \\
\hline $\begin{array}{c}\mathcal{G}_{L} \text { and } \mathcal{G}_{I, L} \\
\text { Viterbi Block Decoding }\end{array}$ & $O\left(N J^{L}(2 L+6)+4 N(1+\varphi)\right)$ & $O\left(N J^{L}(2 L+2)+2 N(1+\varphi)\right)$ \\
\hline $\begin{array}{l}\mathcal{H}_{L} \text { and } \mathcal{H}_{I, L} \\
\mathrm{ZF} \text { Decoding }\end{array}$ & $O(4 N)$ & $O\left(2 N\left(2^{L}+2^{-L}-1\right)\right)$ \\
\hline $\begin{array}{l}\mathcal{U}_{L} \text { and } \mathcal{U}_{I, L} \\
\text { ZF Decoding }\end{array}$ & $O\left(3 N^{2} 2^{-I}+6 N\right)$ & $O\left(N^{2} 2^{1-I}+2 N\right)$ \\
\hline $\begin{array}{c}\mathcal{V}_{L}, \mathcal{W}_{L}, \text { and } \mathcal{W}_{I, L} \\
\text { ZF Decoding }\end{array}$ & $O\left(2 N\left(3-2^{-L}\right)\right)$ & $O\left(2 N\left(L+2^{-L}\right)\right)$ \\
\hline $\begin{array}{l}\text { ZF Demodulation } \\
\text { (uncoded OFDM) }\end{array}$ & $O(4 N)$ & $O(2 N)$ \\
\hline
\end{tabular}

All the constants, sign inversions, and decision thresholds that are independent of data and received symbols are not counted in complexity evaluation. For the $\mathcal{H}_{L}$ and $\mathcal{H}_{I, L}$ encoding and decoding, the constant $2^{-L / 2}$ in all nonzero precoder coefficients is assumed to be absorbed in other system operation and thus not accounted in complexity evaluation. For the Viterbi block decoding of $\mathcal{G}_{L}$ and $\mathcal{G}_{I, L}$-coded OFDM, the $J^{2}$-ary QAM is adopted as the component modulation. For $\mathcal{G}_{L}$ algorithms, $\varphi=1$ if CP-OFDM is considered and $\varphi=0$ otherwise. For $\mathcal{U}_{L}$ encoding and ZF decoding, the complexity results are obtained by setting $I=0$ in the corresponding entries.

\section{APPENDIX}

A) Proof of (21): Using (19) and (20) in (14) yields

$$
\begin{aligned}
\left.\mathcal{S}_{L P}^{B C P}(f)\right|_{\mathcal{G}_{I, L}}= & \frac{P T}{M \sum_{l=0}^{L}\left(\begin{array}{l}
L \\
l
\end{array}\right)^{2}} \sum_{i=0}^{2^{I}-1} \sum_{m=0}^{2^{-I} M-1} \\
& \left|\sum_{l=0}^{L}\left(\begin{array}{l}
L \\
l
\end{array}\right) U_{2^{I}+1}\left(z_{i} ; 0, m+l\right)\right|^{2}
\end{aligned}
$$

Note that $U_{J}(x ; m, n)$ is a generalization of $U(x ; m, n)$ in [10] with $U_{1}(x ; m, n)=U(x ; m, n)$. Analogous to $U(x ; m, n)$, $U_{J}(x ; m, n)$ is attributed with the following two lemmas.

Lemma 1: When $x, n$ and $J$ are given, $U_{J}(x ; m, n)$ satisfies the recursion $U_{J}(x ; m, n)=U_{J}(x ; m-1, n)+U_{J}(x ; m-$ $1, n+1), m=1,2, \ldots$

Lemma 2: When $x, n$ and $J$ are given, $U_{J}(x ; m, n)$ is a linear combination of $U_{J}(x ; 0, n+l)$ for $l=0,1, \ldots, m$ as $U_{J}(x ; m, n)=\sum_{l=0}^{m}\left(\begin{array}{c}m \\ l\end{array}\right) U_{J}(x ; 0, n+l), m=1,2, \ldots$

When $J$ is a positive odd integer, Lemma $l$ is readily proven by induction and used in turn to induce Lemma 2. Using Lemma 2 and $\sum_{l=0}^{L}\left(\begin{array}{c}L \\ l\end{array}\right)^{2}=\left(\begin{array}{c}2 L \\ L\end{array}\right)$, (32) simplifies to (21).

B) Proof of Propositions 3 and 4: For positive integers $L$, $\widetilde{L}$ and $\widetilde{N}$ with both $\widetilde{N}$ and $2^{-L} \widetilde{N}$ being nonnegative integer powers of two and for any real constants $\xi$ and $\varrho$, we define

$$
\begin{gathered}
H_{L, \widetilde{N}}(m, \beta ; \xi, \varrho)=\mid \sum_{\alpha=0}^{2^{L}-1}(-1)^{\sum_{l=1}^{L} \alpha_{l} \beta_{l}}\left[\xi\left(m 2^{L}+\alpha\right)\right. \\
-\varrho]\left.^{-1}\right|^{2}, \\
m \in \mathcal{Z}_{2^{-L} \tilde{N}}, \beta \in \mathcal{Z}_{2^{L}-1}^{+} \\
V_{\widetilde{L}}(u, n ; \xi, \varrho)=\left|\sum_{v=0}^{2^{u}-1}(-1)^{v_{1}}\left[\xi\left(v 2^{\widetilde{L}-u}+n\right)-\varrho\right]^{-1}\right|^{2}, \\
n \in \mathcal{Z}_{2^{\tilde{L}-u}}, u \in \mathcal{Z}_{\widetilde{L}}^{+} \\
W_{L, \widetilde{N}}(u, n ; \xi, \varrho)=\left|\sum_{v=0}^{2^{u}-1}(-1)^{v_{u}}\left[\xi\left(n 2^{u}+v\right)-\varrho\right]^{-1}\right|^{2}, \\
n \in \mathcal{Z}_{2^{-u} \widetilde{N}}, u \in \mathcal{Z}_{L}^{+} .
\end{gathered}
$$

The following Lemmas 3-5 give some useful identities relating these functions which are useful for proving Propositions 3 and 4. Since these identities hold for all admissible $\xi$ and $\varrho$, both arguments are dropped for brevity when referring to the above functions below.

Lemma 3: For $m \in \mathcal{Z}_{2-L \tilde{N}}$ and $u \in \mathcal{Z}_{L-1}^{+}$, $H_{L, \widetilde{N}}\left(m, 2^{L-1}\right)=W_{L, \widetilde{N}}(L, m)$ and (2)

$$
\begin{aligned}
& \sum_{\substack{\beta_{l} \in \mathcal{Z}_{2} \\
u+1 \leq l \leq L}} H_{L, \widetilde{N}}\left(m, 2^{u-1}+\sum_{i=u+1}^{L} \beta_{i} 2^{i-1}\right) \\
= & 2^{L-u} \sum_{\substack{\alpha \in \mathcal{Z}_{2} \\
u+1 \leq l \leq L}} W_{L, \widetilde{N}}\left(u, m 2^{L-u}+\sum_{k=u+1}^{L} \alpha_{k} 2^{k-u-1}\right) .
\end{aligned}
$$

Proof: The first identity can be readily proven. Next, by 
expanding $v=\sum_{l=1}^{u} v_{l} 2^{l-1}$ for all $v_{l} \in \mathcal{Z}_{2}$, (35) can be rewritten as

$$
\begin{aligned}
& W_{L, \tilde{N}}\left(u, m 2^{L-u}+\sum_{k=u+1}^{L} \alpha_{k} 2^{k-u-1}\right) \\
= & \left|F_{L-u}\left(\left\{\alpha_{l}\right\}_{l=u+1}^{L},\left\{\beta_{l}\right\}_{l=u+1}^{L}\right)\right|^{2}
\end{aligned}
$$

for $m \in \mathcal{Z}_{2^{-L} \tilde{N}}$, where we have defined $F_{L-u}\left(\left\{\alpha_{l}\right\}_{l=u+1}^{L},\left\{\beta_{l}\right\}_{l=u+1}^{L}\right) \triangleq(-1)^{\sum_{k=u+1}^{L} \alpha_{k} \beta_{k}} \sum_{\substack{v_{l} \in \mathcal{Z}_{2} \\ 1 \leq l \leq u}}$ $(-1)^{v_{u}}\left[\xi\left(m 2^{L}+\sum_{k=u+1}^{L} \alpha_{k} 2^{k-1}+\sum_{i=1}^{u} v_{i} 2^{i-1}\right)-\varrho\right]^{-1}$. Note that $\left|F_{L-u}\left(\left\{\alpha_{l}\right\}_{l=u+1}^{L},\left\{\beta_{l}\right\}_{l=u+1}^{L}\right)\right|$ is constant for all sets $\left\{\beta_{l}\right\}_{l=u+1}^{L}$. Also, if $\bar{\beta}_{l}=1-\beta_{l}$ denotes the complement of $\beta_{l}$, then

$$
\begin{aligned}
& F_{L-u}\left(\left\{\alpha_{l}\right\}_{l=u+1}^{L},\left\{\beta_{l}\right\}_{l=u+1}^{L}\right)=(-1)^{\alpha_{k}} \\
& \cdot F_{L-u}\left(\left\{\alpha_{l}\right\}_{l=u+1}^{L},\left\{\beta_{u+1}, \ldots, \beta_{k-1}, \bar{\beta}_{k}, \beta_{k+1}, \ldots, \beta_{L}\right\}\right)
\end{aligned}
$$

for $u+1 \leq k \leq L$. These two properties on $F_{L-u}(\cdot, \cdot)$ are useful in the following.

Further, expanding $\alpha=\sum_{l=u+1}^{L} \alpha_{l} 2^{l-1}+\sum_{i=1}^{u} v_{i} 2^{i-1}$ for all $\alpha_{l}, v_{i} \in \mathcal{Z}_{2}$ in (33) gives

$$
\begin{aligned}
& H_{L, \tilde{N}}\left(m, 2^{u-1}+\sum_{i=u+1}^{L} \beta_{i} 2^{i-1}\right) \\
= & \left|\sum_{\substack{\alpha_{l} \in \mathcal{Z}_{2} \\
u+1 \leq l \leq L}} F_{L-u}\left(\left\{\alpha_{l}\right\}_{l=u+1}^{L},\left\{\beta_{l}\right\}_{l=u+1}^{L}\right)\right|^{2}
\end{aligned}
$$

for $m \in \mathcal{Z}_{2^{-L} \tilde{N}}$. This expression expands the left-hand side of (36) as

$$
\begin{aligned}
& \sum_{\substack{\beta_{l} \in \mathcal{Z}_{2} \\
u+1 \leq l \leq L}} H_{L, \widetilde{N}}\left(m, 2^{u-1}+\sum_{i=u+1}^{L} \beta_{i} 2^{i-1}\right) \\
& =\sum_{\substack{\beta_{l} \in \mathcal{Z}_{2} \\
u+1 \leq l \leq L}} \sum_{\substack{\alpha_{l} \in \mathcal{Z}_{2} \\
u+1 \leq l \leq L}} \sum_{\substack{\alpha_{l}^{\prime} \in \mathcal{Z}_{2} \\
u+1 \leq l \leq L}} \\
& F_{L-u}\left(\left\{\alpha_{k}\right\}_{k=u+1}^{L},\left\{\beta_{k}\right\}_{k=u+1}^{L}\right) \\
& \cdot F_{L-u}\left(\left\{\alpha_{k}^{\prime}\right\}_{k=u+1}^{L},\left\{\beta_{k}\right\}_{k=u+1}^{L}\right) \\
& =\sum_{\substack{\alpha_{l} \in \mathcal{Z}_{2} \\
u+1 \leq l \leq L}} \sum_{\substack{\alpha_{l}^{\prime} \in \mathcal{Z}_{2} \\
u+1 \leq l \leq L}} \sum_{\substack{\beta_{1} \in \mathcal{Z}_{2} \\
u+1 \leq l \leq L-1}}\left(1+(-1)^{\alpha_{L}+\alpha_{L}^{\prime}}\right) \\
& \cdot F_{L-u}\left(\left\{\alpha_{k}\right\}_{k=u+1}^{L},\left\{\beta_{u+1}, \ldots, \beta_{L-1}, 0\right\}\right) \\
& \cdot F_{L-u}\left(\left\{\alpha_{k}^{\prime}\right\}_{k=u+1}^{L},\left\{\beta_{u+1}, \ldots, \beta_{L-1}, 0\right\}\right) \\
& =\sum_{\substack{\alpha_{l} \in \mathcal{Z}_{2} \\
u+1 \leq l \leq L}} \sum_{\substack{\alpha_{l}^{\prime} \in \mathcal{Z}_{2} \\
u+1 \leq l \leq L}}\left[\prod_{k=u+1}^{L}\left(1+(-1)^{\alpha_{k}+\alpha_{k}^{\prime}}\right)\right] \\
& \cdot F_{L-u}\left(\left\{\alpha_{k}\right\}_{k=u+1}^{L},\{0,0, \ldots, 0\}\right) \\
& \cdot F_{L-u}\left(\left\{\alpha_{k}^{\prime}\right\}_{k=u+1}^{L},\{0,0, \ldots, 0\}\right)
\end{aligned}
$$

where the second and third equalities are obtained by invoking (38) recursively. Because $1+(-1)^{\alpha_{k}+\alpha_{k}}$ is nonzero only when $\alpha_{k}=\alpha_{k}^{\prime}$, we further have

$$
\begin{aligned}
& \sum_{\substack{\beta_{l} \in \mathcal{Z}_{2} \\
u+1 \leq l \leq L}} H_{L, \tilde{N}}\left(m, 2^{u-1}+\sum_{i=u+1}^{L} \beta_{i} 2^{i-1}\right) \\
= & 2^{L-u} \sum_{\substack{\alpha_{l} \in \mathcal{Z}_{2} \\
u+1 \leq l \leq L}}\left|F_{L-u}\left(\left\{\alpha_{k}\right\}_{k=u+1}^{L},\{0,0, \ldots, 0\}\right)\right|^{2} \\
= & 2^{L-u} \sum_{\substack{\alpha_{l} \in \mathcal{Z}_{2} \\
u+1 \leq l \leq L}}\left|F_{L-u}\left(\left\{\alpha_{k}\right\}_{k=u+1}^{L},\left\{\beta_{k}\right\}_{k=u+1}^{L}\right)\right|^{2}
\end{aligned}
$$

where the second equality stems from the fact that $\left|F_{L-u}\left(\left\{\alpha_{l}\right\}_{l=u+1}^{L},\left\{\beta_{l}\right\}_{l=u+1}^{L}\right)\right|$ is constant for all sets $\left\{\beta_{l}\right\}_{l=u+1}^{L}$. Combining (37) and (39) proves the second identity.

Similarly, the following Lemma 4 can be proven after an analogous manipulation.

Lemma 4: (1) $H_{L, 2^{L}}(0,1)=V_{L}(L, 0)$. (2) For $u \in \mathcal{Z}_{L-1}^{+}$,

$$
\begin{gathered}
\sum_{\substack{\beta_{l} \in \mathcal{Z}_{2} \\
1 \leq l \leq L-u}} H_{L, 2^{L}}\left(0,2^{L-u}+\sum_{i=1}^{L-u} \beta_{i} 2^{i-1}\right) \\
=2^{L-u} \sum_{\substack{\alpha_{l} \in \mathcal{Z}_{2} \\
1 \leq l \leq L-u}} V_{L}\left(u, \sum_{k=1}^{L-u} \alpha_{k} 2^{k-1}\right) .
\end{gathered}
$$

Both Lemmas 3 and 4 are useful for proving the following lemma.

Lemma 5: For all admissible $L$ and $\widetilde{N}$,

$$
\begin{aligned}
& \sum_{m=0}^{2^{-L}} \sum_{\beta=1}^{\tilde{N}^{-1}} H_{L, \widetilde{N}}(m, \beta)=\sum_{u=1}^{L} 2^{L-u} \sum_{n=0}^{2^{-u} \tilde{N}-1} W_{L, \widetilde{N}}(u, n) \\
& \sum_{\beta=1}^{2^{L}-1} H_{L, 2^{L}}(0, \beta)=\sum_{u=1}^{L} 2^{L-u} \sum_{n=0}^{2^{L-u}-1} V_{L}(u, n) .
\end{aligned}
$$

Proof: Invoking Lemma 3, we have

$$
\begin{aligned}
& \sum_{u=1}^{L} 2^{L-u} \sum_{\alpha=0}^{2^{L-u}-1} W_{L, \widetilde{N}}\left(u, m 2^{L-u}+\alpha\right) \\
= & \sum_{u=1}^{L-1} 2^{L-u} \sum_{\substack{\alpha_{l} \in \mathcal{Z}_{2} \\
u+1 \leq l \leq L}} W_{L, \widetilde{N}}\left(u, m 2^{L-u}\right. \\
+ & \left.\sum_{k=u+1}^{L} \alpha_{k} 2^{k-u-1}\right)+W_{L, \widetilde{N}}(L, m) \\
= & \sum_{u=1}^{L-1} \sum_{\substack{\beta_{l} \in \mathcal{Z}_{2} \\
u+1 \leq l \leq L}} H_{L, \widetilde{N}}\left(m, 2^{u-1}+\sum_{i=u+1}^{L} \beta_{i} 2^{i-1}\right) \\
+ & H_{L, \tilde{N}}\left(m, 2^{L-1}\right) \\
= & \sum_{\beta=1}^{L} H_{L, \tilde{N}}(m, \beta)
\end{aligned}
$$


for $m \in \mathcal{Z}_{2^{-L} \tilde{N}}$. Accumulating the results in (43) for all $m$ yields

$$
\begin{aligned}
& \sum_{m=0}^{2^{-L} \tilde{N}-1} \sum_{\beta=1}^{2^{L}-1} H_{L, \tilde{N}}(m, \beta) \\
& =\sum_{m=0}^{2^{-L} \tilde{N}-1} \sum_{u=1}^{L} 2^{L-u} \sum_{\alpha=0}^{2^{L-u}-1} W_{L, \widetilde{N}}\left(u, m 2^{L-u}+\alpha\right) \\
& =\sum_{u=1}^{L} 2^{L-u} \sum_{n=0}^{2^{-u} \widetilde{N}-1} W_{L, \widetilde{N}}(u, n)
\end{aligned}
$$

where the second equality is obtained by the transformation $n=m 2^{L-u}+\alpha$. This proves (41). By invoking Lemma 4, (42) can be similarly proven.

Lemma 5 is general enough to prove Propositions 3 and 4. Specifically, Proposition 3 can be proven by verifying the equivalence between (26) and (27) through the aid of (41) with $\widetilde{N}=N, L \in \mathcal{Z}_{\log _{2} N}^{+}, \xi=1$ and $\varrho=z$. Proposition 4 can be proven in two steps. First, by using (41) with $\widetilde{N}=2^{-I} N$, $L \in \mathcal{Z}_{\log _{2} N-I}^{+}, \xi=2^{I}+1$ and $\varrho=z_{i}$ to compare (24) and (29), $\left.\mathcal{S}_{L P}^{B C P}(f)\right|_{\mathcal{H}_{I, L}}=\left.\mathcal{S}_{L P}^{B C P}(f)\right|_{\mathcal{W}_{I L}}$ can be attained. Second, by using (42) with $L=\log _{2} N-I, \xi=2^{I}+1$ and $\varrho=z_{i}$ to compare (29) and (30), $\left.\mathcal{S}_{L P}^{B C P}(f)\right|_{\mathcal{H}_{I, \log _{2} N-I}}=$ $\left.\mathcal{S}_{L P}^{C P}(f)\right|_{\mathcal{V}_{\log _{2} N-I}}$ can be obtained. Combining both results proves Proposition 4.

\section{REFERENCES}

[1] R. W. Chang, "Synthesis of band-limited orthogonal signals for multichannel data transmission," Bell Syst. Tech. J., vol. 45, pp. 1775-1796, Dec. 1966.

[2] B. R. Saltzberg, "Performance of an efficient parallel data transmission system," IEEE Trans. Commun. Technol., vol. 15, pp. 805-811, Dec. 1967.

[3] B. Hirosaki, "An orthogonally multiplexed QAM system using the discrete Fourier transform," IEEE Trans. Commun., vol. 29, pp. 982 989, July 1981.

[4] C. D. Chung, "Orthogonally-multiplexed orthogonal amplitude modulation family," IEEE Trans. Commun., vol. 50, pp. 415-428, Mar. 2002.

[5] J.A.C. Bingham, "Multicarrier modulation for data transmission: an idea whose time has come," IEEE Commun. Mag., vol. 28, pp. 5-14, May 1990.

[6] X. G. Xia, "Precoded and vector OFDM robust to channel spectral nulls and with reduced cyclic prefix length in single transmit antenna systems," IEEE Trans. Commun., vol. 49, pp. 1363-1374, Aug. 2001.

[7] Z. Wang, S. Zhou, and G. B. Giannakis, "Joint coding-precoding with low-complexity turbo-decoding," IEEE Trans. Wireless Commun., vol. 3, pp. 832-842, May 2004.

[8] M. Debbah, P. Loubaton, and M. de Courville, "Asymptotic performance of successive interference cancellation in the context of linear precoded OFDM systems," IEEE Trans. Commun., vol. 52, pp. 1444-1448, Sept. 2004.

[9] B. Muquet, Z. Wang, G. B. Giannakis, M. de Courville, and P. Duhamel, "Cyclic prefixing or zero padding for wireless multicarrier transmissions?" IEEE Trans. Commun., vol. 50, pp. 2136-2148, Dec. 2002.

[10] C. D. Chung, "Correlatively coded OFDM," IEEE Trans. Wireless Commun., vol. 5, no. 8, pp. 2044-2049, Aug. 2006.

[11] C. D. Chung, "Spectrally precoded OFDM," IEEE Trans. Commun., vol. 54, no. 12, pp. 2173-2185, Dec. 2006.

[12] C. D. Murphy, "Low-complexity FFT structures for OFDM transceivers," IEEE Trans. Commun., vol. 50, pp. 1878-1881, Dec. 2002.

[13] R. Li and G. Stette, "Time-limited orthogonal multicarrier modulation schemes," IEEE Trans. Commun., vol. 43, pp. 1269-1272, Feb./Mar./Apr. 1995.
[14] L. Wei and C. Schlegel, "Synchronization requirements for multi-user OFDM on satellite mobile and two-path Rayleigh fading channels," IEEE Trans. Commun., vol. 43, pp. 887-895, Feb./Mar./Apr. 1995.

[15] A. Vahlin and N. Holte, "Optimal finite duration pulses for OFDM," IEEE Trans. Commun., vol. 44, pp. 10-14, Jan. 1996.

[16] IEEE, Wireless LAN medium access control (MAC) and physical layer (PHY) specifications: Higher speed physical layer (PHY) extension in the $5 \mathrm{GHz}$ band, IEEE Std. 802.11a/D5.0, Apr. 1999.

[17] M. Faulkner, "The effect of filtering on the performance of OFDM systems," IEEE Trans. Veh. Technol., vol. 49, pp. 1877-1884, Sept. 2000.

[18] I. Cosovic, S. Brandes, and M. Schnell, "Subcarrier weighting: a method for sidelobe suppression in OFDM systems," IEEE Commun. Lett., vol. 10, pp. 444-446, June 2006.

[19] S. Brandes, I. Cosovic, and M. Schnell, "Reduction of out-of-band radiation in OFDM systems by insertion of cancellation carriers," IEEE Commun. Lett., vol. 10, pp. 420-422, June 2006.

[20] J. A. C. Bingham, "RFI suppression in multicarrier transmission systems," in Proc. IEEE Global Telecommunications Conference, pp. 10261030, 1996.

[21] W. R. Bennett, Introduction to Signal Transmission. New York: McGraw Hill, 1970.

[22] I. S. Gradshteyn and I. M. Ryzhik, Table of Integrals, Series, and Products, 5th ed. New York: Academic Press, 1994.

[23] K. Hoffman and R. Kunze, Linear Algebra, 2nd ed. Englewood Cliffs, NJ: Prentice Hall, 1971.

[24] J. Tellado, Multicarrier Modulation with Low Peak to Average Power Applications to xDSL and Broadband Wireless. Boston/Dordrecht/London: Kluwer Academic Publishers, 2000.

[25] S. Zhou and G. B. Giannakis, "Finite-alphabet based channel estimation for OFDM and related multicarrier systems," IEEE Trans. Commun., vol. 49, pp. 1402-1414, Aug. 2001.

[26] M. K. Simon, S. M. Hinedi, and W. C. Lindsey, Digital Communication Techniques. Englewood Cliffs, NJ: Prentice Hall, 1994.

[27] M. L. McCloud, "Analysis and design of short block OFDM spreading matrices for use on multipath fading channels," IEEE Trans. Commun., vol. 53, pp. 656-665, Apr. 2005.

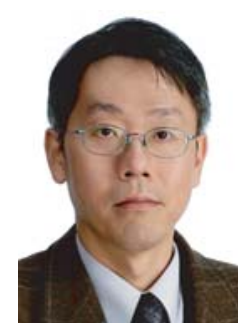

Char-Dir Chung (S'87-M'89-SM'03) received the B.S. degree in electrical engineering from the National Taiwan University, Taipei, in 1983, and the M.S. and Ph.D. degrees in electrical engineering from the University of Southern California, Los Angeles, in 1986 and 1989, respectively.

From 1989 to 1992 he was with the LinCom Corporation, Los Angeles, where he worked on analytical and simulation modeling of scientific and military satellite communication systems. From 1992 to 2005 he was with the National Central University, Chung-Li, Taiwan, ROC, first as an Associate Professor, then as a Professor since 1996. At the National Central University, he founded the Advanced Communications Laboratory in 1998, the Graduate Institute of Communications Engineering in 2000 and the Department of Communications Engineering in 2003, and was the founding heads of these organizations. Since 2005 he has been on the faculty at the National Taiwan University, Taipei, Taiwan, ROC, where he is now a Professor of the Graduate Institute of Communications Engineering and the Department of Electrical Engineering. His current research interests are in digital modulation theory, wireless communications, and spread spectrum communications. He has published more than 60 journal and conference papers and holds two patents in these areas.

Dr. Chung received the Group Achievement Award from the National Aeronautics and Space Administration, USA, in 1991, the Young Scientists Award from the International Union of Radio Science in 1993, the annual Research Award from the National Science Council, ROC, in 1992 and from 1994 to 2001, the Kentucky Colonel grade from the Commonwealth of Kentucky, USA, in 2003, and the FORMOSAT-2 Satellite Project Award from the National Space Center, ROC, in 2005. He served as the Chairman of IEEE Information Theory Society, Taipei Chapter, from 1997 to 1999, and the Secretary of IEEE Taipei Section since 2007. From 2000 to 2004 he was an Editor for the Journal of the Chinese Institute of Electrical Engineering, and since 2003 he has been an Editor for the Electrical Engineering Magazine of the same organization. Since 2004, he has been appointed the Chairman of the Wireless System Group of the National Science and Technology Program for Telecommunications, ROC, and the Chairman of the Taiwan Broadband Wireless Communications Industry Alliance, ROC. In the past decade, he has also actively served as a technical referee of numerous industrial product and technology development projects for several ROC government agencies. 